\title{
3DFaceFill: An Analysis-By-Synthesis Approach to Face Completion
}

\author{
Rahul Dey Vishnu Naresh Boddeti \\ Michigan State University \\ \{deyrahul, vishnu\}emsu.edu
}

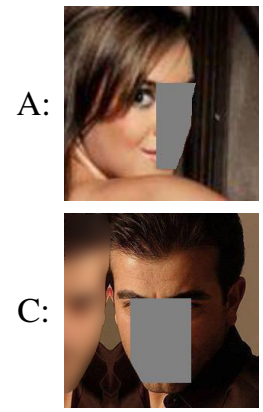

(a) Input

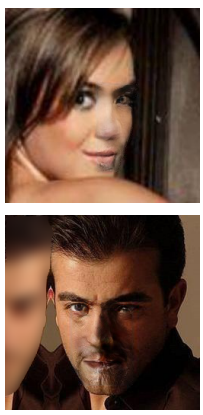

(b) DeepFillv2

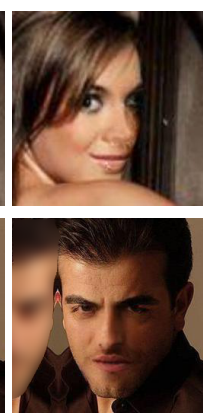

(c) PICNet

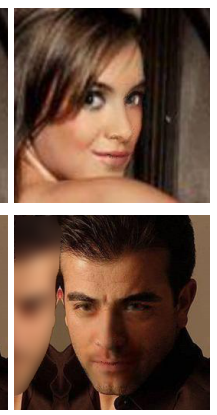

(d) 3DFaceFill

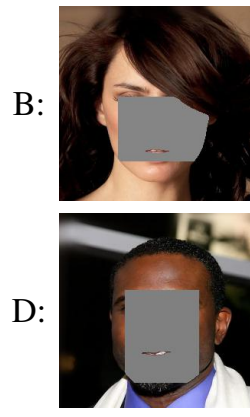

(a) Input

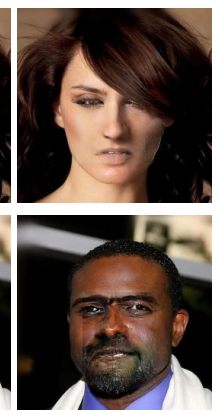

(b) DeepFillv2

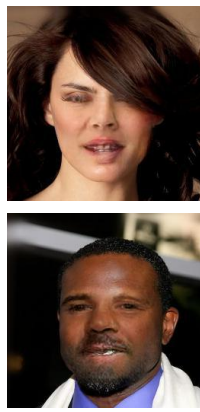

(c) PICNet

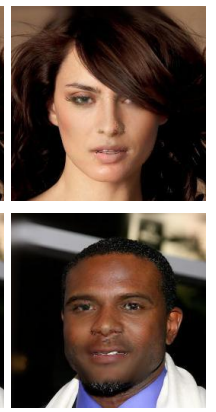

(d) 3DFaceFill

Figure 1: Inpainting of face images under diverse conditions by 3DFaceFill and existing approaches. By modeling the image formation process $3 \mathrm{DFaceFill}$ is able to generate more geometrically consistent and photorealistic completions across diverse scenarios such as non-frontal poses (A), light and dark complexions (B,D), non-uniform facial illumination (e.g. illumination is different on two sides of the nose in $\mathrm{C}$ ) and in cases where the baselines tend to distort face components (e.g. nose in $\mathrm{B})$.

\section{Abstract}

Existing face completion solutions are primarily driven by end-to-end models that directly generate $2 D$ completions of $2 D$ masked faces. By having to implicitly account for geometric and photometric variations in facial shape and appearance, such approaches result in unrealistic completions, especially under large variations in pose, shape, illumination and mask sizes. To alleviate these limitations, we introduce 3DFaceFill, an analysis-by-synthesis approach for face completion that explicitly considers the image formation process. It comprises three components, (1) an encoder that disentangles the face into its constituent $3 D$ mesh, 3D pose, illumination and albedo factors, (2) an autoencoder that inpaints the UV representation of facial albedo, and (3) a renderer that resynthesizes the completed face. By operating on the UV representation, 3DFaceFill affords the power of correspondence and allows us to naturally enforce geometrical priors (e.g. facial symmetry) more effectively. Quantitatively, 3DFaceFill improves the stateof-the-art by up to $4 d B$ higher PSNR and $25 \%$ better LPIPS for large masks. And, qualitatively, it leads to demonstrably more photorealistic face completions over a range of masks and occlusions while preserving consistency in global and component-wise shape, pose, illumination and eye-gaze.

\section{Introduction}

End-to-end image completion methods i.e., models that generate 2D completions directly from 2D masked images, have witnessed remarkable progress in recent years. These approaches rely primarily on architectural advances in neural network designs to implicitly account for photometric and geometric variations in image appearance. And even those that explicitly include scene geometry in their formulation do so largely in 2D. Consequently, object-based image completions from such methods often suffer from poor photorealism, especially under large variations in pose, shape, illumination of objects in the image and the inpainting mask. For example, in the context of faces, Fig. 1 shows face images having extreme poses (1)A), illumination variations across the face (1.C) and diverse appearances and shapes. Current state-of-the-art methods such as DeepFillv2 [46] and PICNet [51], both of which operate end-toend on $2 \mathrm{D}$ image representations, often fail in preserving facial symmetry and the variations of the aforementioned factors (pose, illumination, texture, shape) while inpainting.

Several attempts have been made to customize generic image inpainting solutions for structured objects such as faces. General image inpainting approaches typically employ a CNN autoencoder as the inpainter and train it using a combination of photometric and adversarial losses 


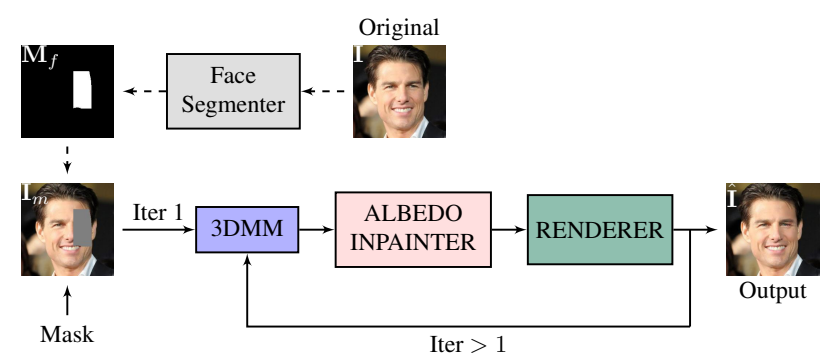

Figure 2: Overview: 3DFaceFill is an iterative inpainting approach where the masked face is disentangled into its 3D shape, pose, illumination and partial albedo by the 3DMM module, following which the partial albedo is inpainted and finally the completed image is rendered. During inference (only), the completed image is fed back through the whole pipeline in subsequent iterations, while using the initial mask for albedo inpainting. During training, a pre-trained model segments the image into face, hair and background for constraining the mask to lie only on the face. This segmentation is optionally used during inference if necessary.

[14, 27, 45, 51]. Face specific completion methods [20, 35] employ additional losses such as landmark loss, perceptual loss and face parsing loss. However, these approaches still do not account for all factors in the image formation process like illumination and pose variations and as such fail to effectively impose geometric priors such as facial symmetry. Moreover, the implicit enforcement of geometric priors is still done in 2D as opposed to in 3D. This is a significant limitation as faces are inherently symmetric 3D objects and their projections on 2D images are often affected by the aforementioned factors of pose, illumination, shape etc.

In contrast to the foregoing, this paper advocates for an analysis-by-synthesis approach for face completion that explicitly accounts for the 3D structure of faces i.e., shape and albedo, and image formation factors i.e., pose and illumination. The key insight of our solution is that performing face completion on the UV representation, as opposed to the 2D pixel representation, allows us to effectively leverage the power of correspondence and ultimately lead to geometrically and photometrically accurate face completion (see Fig 11). Our approach (see Fig. 2), dubbed 3DFaceFill, comprises of three components that are iteratively executed. First, the masked face image is disentangled into its constituent geometric and photometric factors. Second, an autoencoder performs inpainting on the UV representation of facial albedo. Lastly, the completed face is re-synthesized by a differentiable renderer. Our specific contributions are:

- We propose 3DFaceFill, a simple yet very effective face completion model that explicitly disentangles photometric and geometric factors and perform inpainting in the UV representation of facial albedo while preserving the associated facial shape, pose and illumination.

- We propose a 3D symmetry-aware network architecture and a symmetry loss for the inpainter to propagate albedo features from the visible to symmetric masked regions of the UV representation. Enforcing the symmetry prior in 3D, as opposed to $2 \mathrm{D}$, allows $3 \mathrm{DF}$ aceFill to more effectively leverage and preserve facial symmetry while inpainting.

- Given our trained model, we propose a simple refinement process at inference by iteratively reprocessing the face completion through the model. This process enables us to address the "chicken-and-egg" problem of simultaneously inferring both the photometric and geometric factors and completion of the face from a masked image. The procedure is especially effective for heavily masked faces, improving the PSNR by up to $1 \mathrm{~dB}$.

- Extensive benchmarking on several datasets and unconstrained in-the-wild images results in 3DFaceFill producing photorealistic and geometrically consistent face completions over a range of masks and real occlusions, especially in terms of pose, lighting, and attributes such as eyegaze and shape of nose along with a quantitative improvement of upto 4dB PSNR and 25\% in LPIPS [49].

\section{Related Work}

Image Inpainting: Earlier image inpainting approaches [1, 2, 6, 12] used diffusion or patch based methods to fill in the missing regions. This produced sharp results but often lacked semantic consistency. Recent techniques employ a CNN autoencoder along with a GAN loss to generate semantically consistent and realistic completions [14, 27, 44]. More recent methods focus on architectural enhancements to improve inpainting for variable and free form masks. These include a more refined discriminator in PatchGAN [15], contextual attention by DeepFill [45] and gated convolutions [23, 46]. In contrast, we adopt vanilla $\mathrm{CNN}$ architectures and instead rely on a more accurate analysis-bysynthesis modeling approach. Recently, Zheng et al. [51] generated multiple completions by sampling from a conditional distribution. Though this is a topic of interest, it is orthogonal to the goals of the current paper.

Face Completion: Face completion is a more challenging variant of image completion because of the complexity and diversity of faces. To address this, many approaches impose additional geometric and photometric priors in the form of face related losses [4, 20, 21, 35, 47, 50]. A recent approach called DSA [52] uses oracle-learned attention maps and component-wise discriminators to generate highfidelity completions. While it often generates photorealistic completions in well-lit frontal faces, it still relies on implicitly learned priors which are insufficient to enforce correct geometry in challenging poses and illuminations. All these approaches rely on novel architectural advances and loss functions while 3DFaceFill focuses on more explicit and precise modeling of the image-formation process. 

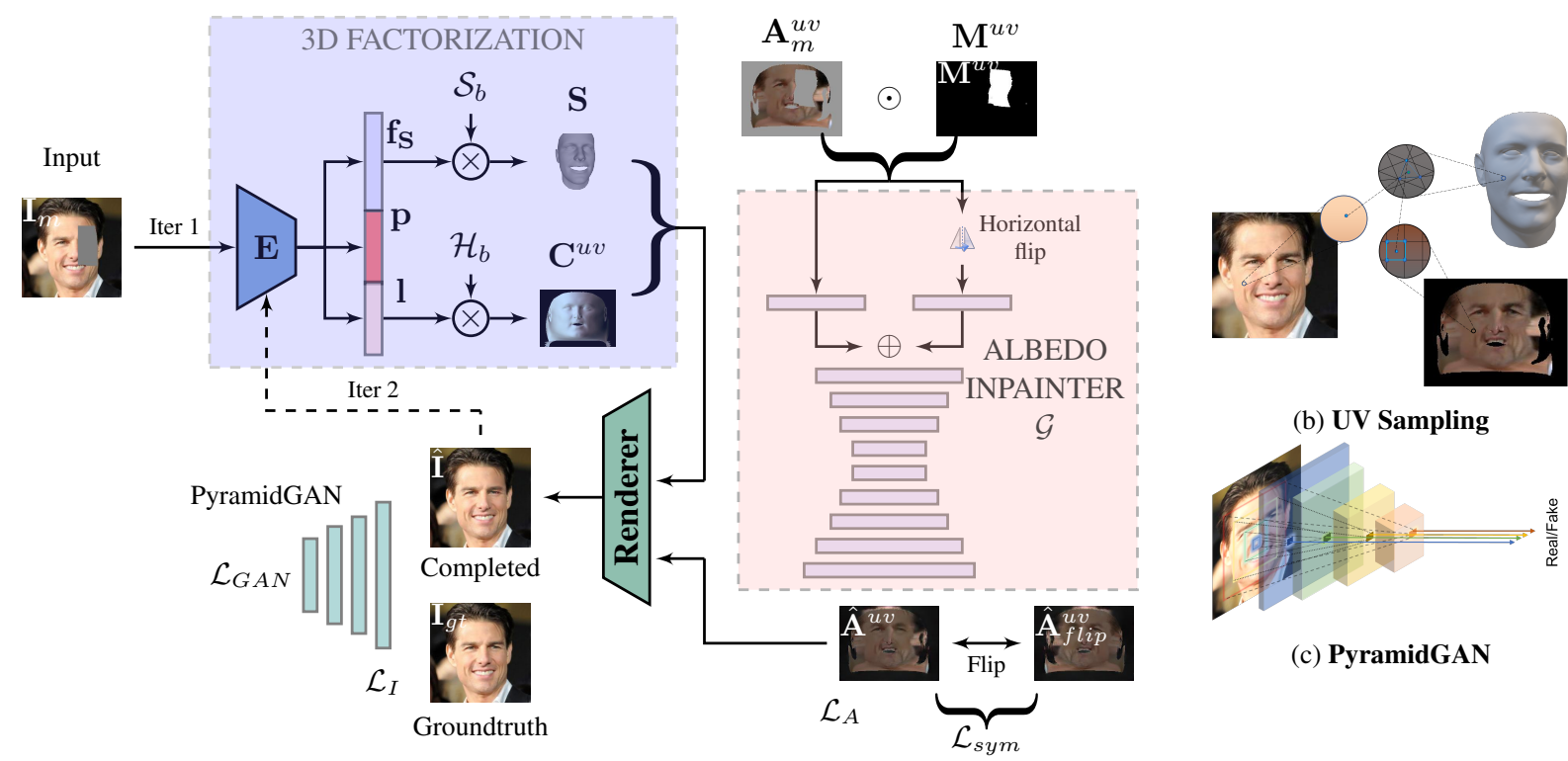

(b) UV Sampling

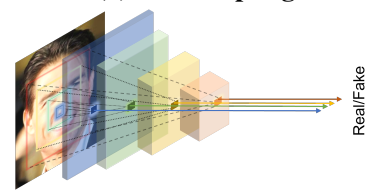

(c) PyramidGaN

(a) Architecture

Figure 3: (a) Architecture: Given a masked face $\mathbf{I}_{m}$, the 3DMM encoder extracts its shape $\mathbf{f}_{\mathbf{S}}$, pose $\mathbf{p}$ and illumination $\mathbf{l}$ parameters, from which we obtain the full shape $\mathbf{S}$ and shade $\mathbf{C}^{u v}$ by linear combination of the corresponding bases. Then a partial albedo $\mathbf{A}_{m}^{u v}$ is obtained by first re-projecting the 3D mesh onto the masked image to obtain the UV-texture, as shown in (b) and then removing the shade from it $\mathbf{A}_{m}^{u v}=\mathbf{T}_{m}^{u v} \oslash \mathbf{C}^{u v}$. Finally, the albedo inpainter $\mathcal{G}$ completes the partial albedo as $\hat{\mathbf{A}}^{u v}$, conditioned on the UV-mask $\mathbf{M}{ }^{u v}$, which is rendered along with the estimated shape, pose and shade to obtain the completed image $\hat{\mathbf{I}}$. To generate photorealistic completion, the completed and groundtruth images are evaluated by the proposed (c) PyramidGAN discriminator. (b) UV Sampling: 3D mesh is projected onto the face image to obtain per vertex RGB values $\mathbf{T}_{\mathbf{v}}(x, y, z)$. Each mesh face triangle $\mathbf{t}=\left(\mathbf{v}_{\mathbf{1}}, \mathbf{v}_{\mathbf{2}}, \mathbf{v}_{\mathbf{3}}\right)$ is mapped to a particular pixel in the UV space $\mathbf{T}_{m}^{v}(t) \rightarrow \mathbf{T}_{m}^{u v}(u, v)$ which allows us to sample the UV texture using barycentric interpolation.

Concurrently, Deng et al. [7] completed self-occluded UV texture to synthesize new face views. This assumes that the full face image and at least half of the UV texture is always visible. In contrast, we go beyond self-occlusion and instead, perform 3D factorization on the masked face and complete its albedo for masked face completion. Furthermore, since texture is not always symmetric due to illumination variations, [7] needs synthetically completed texture maps for training; whereas our model performs completion on albedo which is further disentangled from both geometry as well as illumination allowing us to effectively enforce symmetry prior, without needing synthetically completed UV-maps for training, as it bears out in our experiments. A few recent works have also attempted to leverage symmetry for face completion [19,48]. However, these approaches employ complex symmetry registration operations, which require huge computational resources; moreover these operations are often susceptible to large geometric variations.

\section{Approach}

In this section, we first present an overview of our proposed 3D face completion approach (dubbed 3DFaceFill) followed by the details of each component. As shown in Fig. 2, 3DFaceFill has three components: a 3DMM en- coder, an albedo completion module and a renderer. Given a masked face, 3DFaceFill first resolves it into its constituent 3D shape, pose and illumination using the 3DMM encoder (Fig. 3). Then, we obtain the partial facial texture in the UV-domain by re-projecting the mesh onto the input image (Fig. 3b). We further remove the shading component to obtain an illumination-invariant partial albedo. The inpainter completes the partial albedo using symmetric and learned priors. Finally, the renderer combines the inpainted albedo with the estimated 3D factors to obtain the completed face. As a natural extension of the proposed approach, we use $3 \mathrm{D}$ factorization and completion in a complimentary way to further improve completion iteratively.

\subsection{D Factorization}

Existing face image completion approaches directly operate on 2D, which makes it non-trivial to enforce strong 3D geometric and photometric priors. This leads to poor face completion in challenging conditions of poses, geometry, lighting, etc. This motivates us to adopt explicit 3D factorization of face images to disentangle the appearance and geometric components, to enable robust completion.

Essentially, the 3D factorization module is an inverse renderer $\Phi: \mathbf{I} \rightarrow(\mathbf{S}, \mathbf{p}, \mathbf{l}, \mathbf{A})$ that resolves a $2 \mathrm{D}$ face $\mathbf{I}$ 
into its constituent shape $\mathbf{S} \in \mathbb{R}^{3}$, pose $\mathbf{p}$, illumination $\mathbf{l}$ and albedo A. Various 3DMM approaches like [3, 8, 9] can be a natural fit for this. However, they are not real time, leaving learning based 3D reconstruction approaches [32, 34, 36, 39, 42] as the obvious choices. While any of these approaches can potentially be used in our approach, for the purpose of this work, we adopt a simplified version of the nonlinear 3DMM presented by Tran et al. [38].

The 3D factorizaiton module consists of a 3DMM encoder and an albedo decoder (used only during training). The encoder $\mathcal{E}$ first resolves the image $\mathbf{I}$ in to its shape, albedo and illumination coefficients $\left(\mathbf{f}_{\mathbf{S}}, \mathbf{f}_{\mathbf{A}}, \mathbf{l}\right)$ and pose $\mathbf{p}=(s, \mathbf{R}, \mathbf{t})$. Using the shape coefficients, we obtain the full shape $\mathbf{S}$ by linear combination with the Basel Face Model's (BFM) bases [28]. Similarly, we combine the illumination coefficients linearly with the spherical harmonics ( $\mathrm{SH})$ bases $\mathbf{H}_{b}$ [30] to obtain the surface shading $\mathbf{C}^{u v}$ (we assume Lambertian surface reflectance). The decoder $\mathcal{D}_{\mathbf{A}}$ maps the albedo coefficients into the full UV-albedo $\mathcal{D}_{\mathbf{A}}: \mathbf{f}_{\mathbf{A}} \rightarrow \mathbf{A}^{u v}$, which is then multiplied with the shade to obtain the texture $\mathbf{T}^{u v}=\mathbf{A}^{u v} \odot \mathbf{C}^{u v}$. A differentiable renderer $\mathcal{R}$ [38] then reprojects the estimated 3D factors into image $\mathbf{I}_{\text {ren }}$ using the Z-buffer technique:

$$
\mathbf{I}_{\text {ren }}=\mathcal{R}(\mathbf{S}, \mathbf{A}, \mathbf{p}, \mathbf{l})
$$

We train the module using masked images for robustness to partial inputs. For further details, refer the supplement.

\subsection{Albedo Completion Module}

Architecturally, our albedo completion module is similar to other adversarially trained image-completion autoencoders [20, 27, 45]. However, ours has the unique advantage of being solely focused on recovering the missing albedo, which has been disentangled from other variations in shape, pose and illumination through $3 \mathrm{D}$ factorization and is largely symmetric in its UV-representation. UVGAN [7] performs a similar completion of self-occluded UVtexture extracted from fully-visible face images. However, because of the entangled illumination, they don't use symmetry and need a synthetically completed texture map for supervision, whereas we use symmetry as self-supervision.

To this end, we discard the soft albedo obtained from the 3DMM albedo decoder and instead obtain the more realistic partial albedo from the input image in the UV space. This is done in two steps: first, we reproject the obtained 3D mesh onto the face image and use bilinear interpolation to sample the per-vertex texture (see Fig. 3b):

$$
\mathbf{T}_{m}^{\mathbf{v}}(x, y, z)=\sum_{\substack{p \in\{\lfloor x\rfloor,\lceil x\rceil\} \\ q \in\{\lfloor y\rfloor,\lceil y\rceil\}}} \mathbf{I}_{m}^{p, q}(1-|x-p|)(1-|y-q|)
$$

Then, we map the sampled partial texture $\mathbf{T}_{m}^{\mathbf{v}}$ onto the UV space using barycentric interpolation on the predefined mesh-to-uv mappings $\mathbf{T}_{m}^{\mathbf{v}}\left(\mathbf{v}_{1}, \mathbf{v}_{2}, \mathbf{v}_{3}\right) \rightarrow \mathbf{T}_{m}^{u v}(u, v)$. From the texture, we obtain the partial albedo by simply removing the estimated shade: $\mathbf{A}_{m}^{u v}=\mathbf{T}_{m}^{u v} \oslash \mathbf{C}^{u v}$, where $\oslash$ is the element-wise division operation. We perform similar operations to unwarp the mask $\mathbf{M}$ on-to the $\mathbf{U V}$-space as $\mathbf{M}^{u v}$.

We use a U-Net [31] based autoencoder $\mathcal{G}$ to complete the partial albedo conditioned on the input mask, $\mathcal{G}:\left(\mathbf{A}_{m}^{u v}, \mathbf{M}^{u v}\right) \rightarrow\left(\hat{\mathbf{A}}^{u v}, \sigma^{u v}\right)$, where $\hat{\mathbf{A}}^{u v}$ is the completed albedo and $\sigma^{u v}$ is the uncertainty of completion. In order to leverage the bilateral symmetry of the UV facial albedo as an attention map, we modify the U-Net architecture (henceforth referred to as Sym-UNet). This is specially helpful since we do not have access to the full groundtruth albedo maps for training. To do so, we split the first convolution layer $f_{1: 2 c}$ into two parts: $f_{1,1: c}$ and $f_{2, c+1: 2 c}$ with equal number of output channels $c$ (see Fig. 2). The first filter operates on the input albedo as such $\mathbf{h}_{1}=f_{1}\left(\mathbf{A}_{m}^{u v}\right)$. The second, instead, operates on the horizontally flipped albedo $\mathbf{h}_{2}=f_{2}\left(h f l i p\left(\mathbf{A}_{m}^{u v}\right)\right)$. We then concatenate the activations $\mathbf{h}_{1}$ and $\mathbf{h}_{2}$ from these two filters and pass it through the rest of the network. During training, the first filter learns to extract features from the visible parts of the albedo while the second filter learns to extract features corresponding to the symmetrically opposite visible parts to apply on the occluded regions (see Sec. 3.2 in the supplementary).

A naive approach of doing so, however, results in artifacts from the symmetrical counterparts to appear on the visible regions, making the network convergence difficult. Instead, we use gated convolutions [46] (in all but the final layer), to ensure that such symmetric features are only transferred to the masked regions and do not create artifacts on the visible regions. We use group normalization [43] and ELU activation [5] for all the feature layers and the final output is simply clipped between -1 and 1. We then render the completed albedo $\hat{\mathbf{A}}^{u v}$, along with the estimated shape, pose and illumination to obtain a completed image $\hat{\mathbf{I}}$ using eqn. 1 . Finally, we simply blend the input and completed images to obtain the output image: $\mathbf{I}_{\text {out }}=\mathbf{I} \odot(1-\mathbf{M})+\hat{\mathbf{I}} \odot \mathbf{M}$.

PyramidGAN Discriminator: To generate sharp and semantically realistic completions, we use a multi-scale PatchGAN discriminator [33,41], which we refer to as the PyramidGAN. The PyramidGAN evaluates the final output $\mathbf{I}_{\text {out }}$ at multiple locations and scales ranging from coarse and global to fine and local (refer to Fig. 3c). Features from each $l$-th downsampling layer of the PyramidGAN $\mathcal{D}_{l}$ are used to evaluate an average hinge loss [16,46] for that layer. We then compute the average loss across all the layers as the total loss, thus giving equal weightage to each scale:

$\mathcal{L}_{\mathcal{G}}=-\mathbb{E}_{p(z)}\left[\mathbb{E}_{l \in L}\left[\mathcal{D}_{l}(\mathcal{G}(z)]\right]\right.$

$\mathcal{L}_{\mathcal{D}}=\mathbb{E}_{x}\left[\mathbb{E}_{l \in L}\left[\mathbf{1}-\mathcal{D}_{l}(x)\right]_{+}\right]+\mathbb{E}_{p(z)}\left[\mathbb{E}_{l \in L}\left[\mathbf{1}+\mathcal{D}_{l}(\mathcal{G}(z)]_{+}\right]\right.$, 
Training Losses: We train the albedo completion module with the following total loss:

$$
\mathcal{L}=\lambda_{1} \mathcal{L}_{A}+\lambda_{2} \mathcal{L}_{I}+\lambda_{3} \mathcal{L}_{\text {sym }}+\lambda_{4} \mathcal{L}_{G A N}+\lambda_{5} \mathcal{L}_{g p},
$$

where $\mathcal{L}_{A}=\mathcal{L}_{\sigma}\left(\left\|\hat{\mathbf{A}}^{u v}-\hat{\mathbf{A}}_{g t}^{u v}\right\|_{1}, \sigma^{u v}\right)$ and $\mathcal{L}_{I}=\mathcal{L}_{\sigma}(\| \hat{\mathbf{I}}-$ $\left.\mathbf{I}_{g t} \|_{1}, \sigma\right)$ are the pixel losses for the albedo and the image, respectively, $\mathcal{L}_{\text {sym }}$ is the symmetry loss, $\mathcal{L}_{G A N}$ is the GAN loss given in eqn. 2 and $\mathcal{L}_{g p}$ is the WGAN-GP loss as described in [11]. The albedo symmetry loss is carefully applied on the masked regions whose symmetric counterparts are visible, to supplement as supervised attention:

$$
\mathcal{L}_{\text {sym }}=\mathcal{L}_{\sigma}\left(\left(\mathbf{1}-\mathbf{M}^{u v}\right) \mathbf{M}_{f l i p}^{u v} \odot\left\|\hat{\mathbf{A}}^{u v}-\hat{\mathbf{A}}_{f l i p}^{u v}\right\|_{1}, \sigma^{u v}\right)
$$

Here, $\mathcal{L}_{\sigma}(\mathbf{x}, \sigma)=\frac{1}{D} \sum_{i} \frac{1}{2} x_{i} \exp \left(-\sigma_{i}\right)+\frac{\sigma_{i}}{2}$ is the aleatoric uncertainty loss [17]. The loss coefficients are set to have similar magnitude for all the loss components. In this paper, the goal is to show the efficacy of explicit 3D consideration on the geometric and photometric accuracy of face completion. So, we withhold from using attention or face specific losses [20:45: $46,51,52]$ and leave them as future add-ons.

Iterative Refinement: 3D factorization is an important first step of our proposed approach, which itself leads to robust face completion in cases where $2 \mathrm{D}$ based methods fail. To make the 3D factorization itself robust to partial images, we train the 3DMM encoder on face images with randomly sized and randomly located masks. However, there is scope to further improve upon this and leverage the full power of our proposed two-step approach. To do this, we adopt a simple iterative refinement technique where face completion leads to improved 3D factorization and vice versa, as shown in Fig. 2 During inference, the masked face is used to distill the 3D factors in the first iteration; while in the next iteration, the completed face itself forms the input for $3 \mathrm{D}$ analysis. This leads to iteratively refined $3 \mathrm{D}$ analysis (specially the $3 D$ pose) as well as face completion. Though one can repeat the iterative step many times, we experimentally found that two such iterations are usually sufficient.

\section{Experimental Evaluation}

Datasets: We evaluate the proposed 3DFaceFill on the CelebA [24] and CelebA-HQ [18] datasets. We use 80\% split for training and $20 \%$ for evaluation. Further, to evaluate the robustness and generalization performance, we do a cross-dataset evaluation on the pose and illumination varying images from the MultiPIE [10] dataset and 50 in-thewild face images downloaded from the interne ${ }^{1}$

Implementation Details: We train both the 3D factorization and the completion modules independently using the Adam optimizer with a learning rate of $10^{-4}$. We first

\footnotetext{
${ }^{1}$ Source: https://unsplash.com/s/photos/face
}

train the 3DMM module on the 300W-3D [53] and the CelebA [24] datasets. Once the 3DMM encoder is trained, we freeze it and use it to train the completion module on the CelebA [24] dataset for 30k iterations. We generate random rectangular masks of varying sizes and locations, and constrain them to lie in the segmented face region (Fig. 2). We provide further details on implementation and computational analysis in the supplementary.

Baselines: To evaluate the efficacy of 3DFaceFill, we perform qualitative and quantitative comparison against baselines such as GFC [20], SymmFCNet [19], DeepFillv2 [45, 46] and PICNe [2] [51]. We use the publicly available pretrained face models for DeepFillv2 [46], PICNet [51] and SymmFCNet [19]. For GFC [20], the pretrained model was not trained on the same crop and alignment as ours, so we train it from scratch using their source code. Due to the absense of extensive results, we present additional evaluation against baselines that do not provide source codes or pre-trained models in the supplementary, using a small set of results obtained from the corresponding authors.

\subsection{Results}

Quantitative Evaluation: In addition to the typically used PSNR and SSIM metrics, we report LPIPS [49], which is more suitable for image completion. Table 4 reports the overall values of these metrics across all image-mask pairs for each dataset. Overall 3DFaceFill improves PSNR by $2 \mathrm{~dB}-3 \mathrm{~dB}$ and LPIPS by $5-10 \%$ over the closest baselines. In addition, for all the methods, we report PSNR and LPIPS as a function of mask to face area ratio $\left(\frac{\# \text { MaskPixels }}{\# \text { FacePixels }}\right)$ in Fig. 4a, 4b and 4c for the CelebA, CelebA-HQ and MultiPIE datasets, respectively. We make the following observations: (1) Across all the datasets, 3DFaceFill achieves significantly better PSNR and LPIPS across all mask ratios. (2) Among the baselines, PIC [51] and DeepFillV2 [46] perform comparably with the former being slightly better in terms of LPIPS. (3) The effectiveness of 3DFaceFill over the baselines is more apparent as larger parts of the face are to be completed i.e., as the mask ratio increases. (4) On the CelebA dataset [24], the improvement ranges from $\sim 2 \mathrm{~dB}$ PSNR for $0-10 \%$ mask ratio to $\sim 4 \mathrm{~dB}$ PSNR for $60-80 \%$ mask ratio. In terms of LPIPS, the improvement ranges from $5 \%$ for $0-10 \%$ mask ratio to $25 \%$ for $60-90 \%$ mask ratio. Similar trends are seen across the CelebAHQ [18] and MultiPIE [10] datasets too. These results confirm our hypothesis that explicitly modeling the image formation process leads to significantly better face completion. We provide addtional quantitative comparisons against PConv [23], DSA [52] and UVGAN [7] in the supplementary since these results are based on a limited number of author-provided completions in the absense of source codes.

\footnotetext{
${ }^{2}$ Following author guidelines, we sample top 10 completions ranked by its discriminator and chose the one closest to the groudtruth for evaluation.
} 


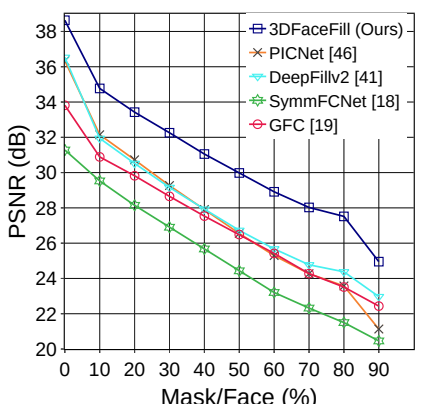

(a) CelebA dataset [24]
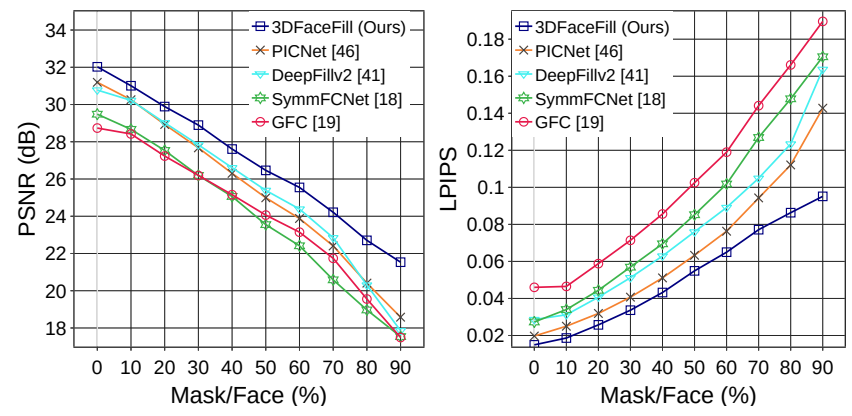
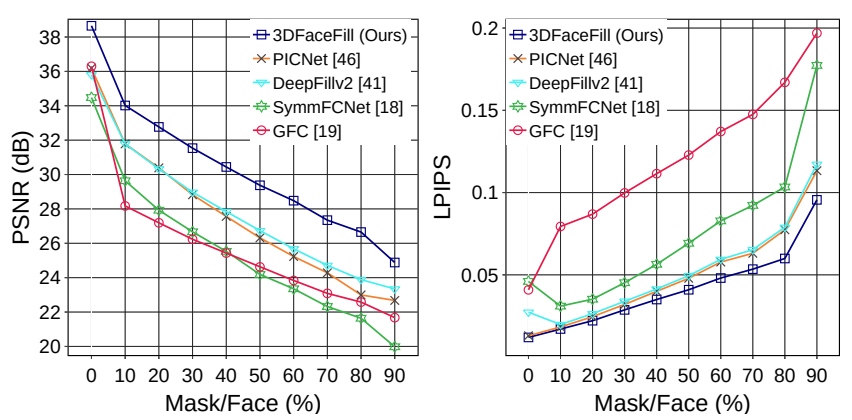

(b) CelebA-HQ dataset $[18$

\begin{tabular}{|c|c|c|c|c|c|c|}
\hline Dataset & Metric & \begin{tabular}{l|l}
$\overline{\text { GFC }}$ & $\overline{20}$
\end{tabular} & SymmFC $[\overline{19}]$ & $\begin{array}{ll}\text { DeepFill } & \overline{46}\end{array}$ & PIC 51$]$ & 3DFaceFill \\
\hline \multirow{3}{*}{ CelebA } & PSNR $(\uparrow)$ & $27.02 \overline{98}$ & 25.8817 & 28.2097 & 28.1262 & 30.4917 \\
\hline & $\operatorname{SSIM}(\uparrow)$ & 0.9257 & 0.9273 & 0.9356 & 0.9424 & 0.9521 \\
\hline & LPIPS $(\downarrow)$ & 0.1134 & 0.0537 & 0.0499 & 0.0362 & 0.0326 \\
\hline \multirow{3}{*}{ CelebAHQ } & PSNR $(\uparrow)$ & 25.5836 & 25.6203 & 27.9885 & 27.7020 & 29.9398 \\
\hline & $\operatorname{SSIM}(\uparrow)$ & 0.8895 & 0.9232 & 0.9311 & 0.9380 & 0.9492 \\
\hline & LPIPS $(\downarrow)$ & 0.1076 & 0.0535 & 0.0394 & 0.0376 & 0.0365 \\
\hline \multirow{3}{*}{ MultiPIE } & PSNR $(\uparrow)$ & 25.3805 & 25.1280 & 26.8225 & 26.5574 & 28.7515 \\
\hline & $\operatorname{SSIM}(\uparrow)$ & 0.9127 & 0.9266 & 0.9391 & 0.9397 & 0.9553 \\
\hline & LPIPS $(\downarrow)$ & 0.0798 & 0.0645 & 0.0577 & 0.0472 & 0.0436 \\
\hline
\end{tabular}

(d) Average metrics across all masks.

(c) MultiPIE dataset 10

Figure 4: Quantitative Evaluation: We perform face completion over (a) CelebA [24], (b) CelebA-HQ [18] and (c) MultiPIE [10] datasets across a range (0-90\%) of mask to face area ratios and evaluate the PSNR and LPIPS [49] metrics. In addition, we report the overall metrics across all mask-to-face are ratios in Table (d). 3DFaceFill consistently outperforms the baselines across all the datasets and mask ratios.

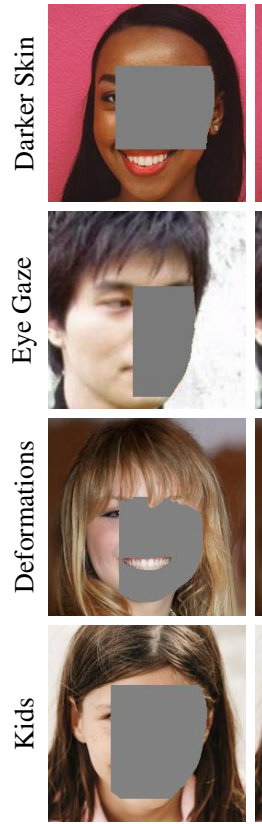

Input
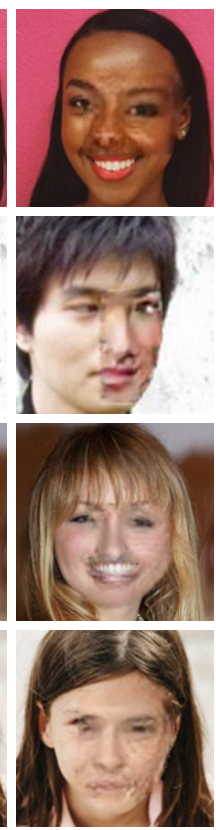

GFC [20]
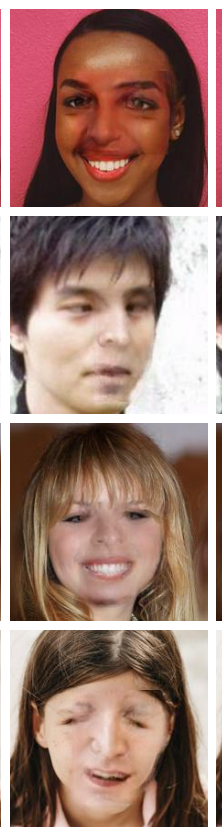

SymmFCNet [19]
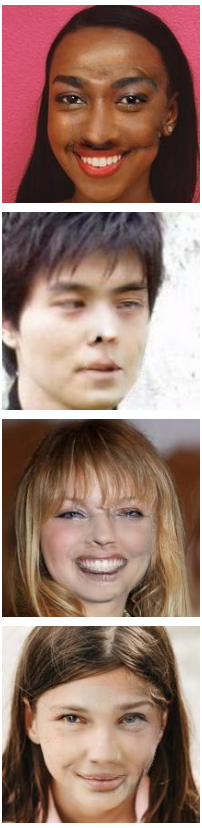

DeepFill [46]
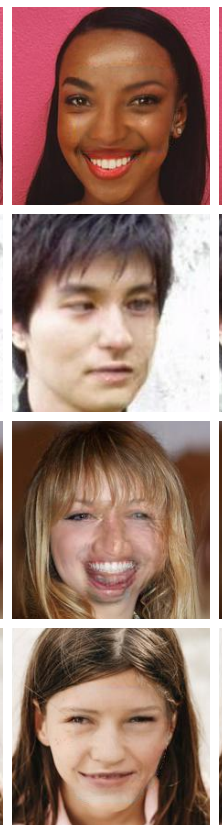

PIC [51]
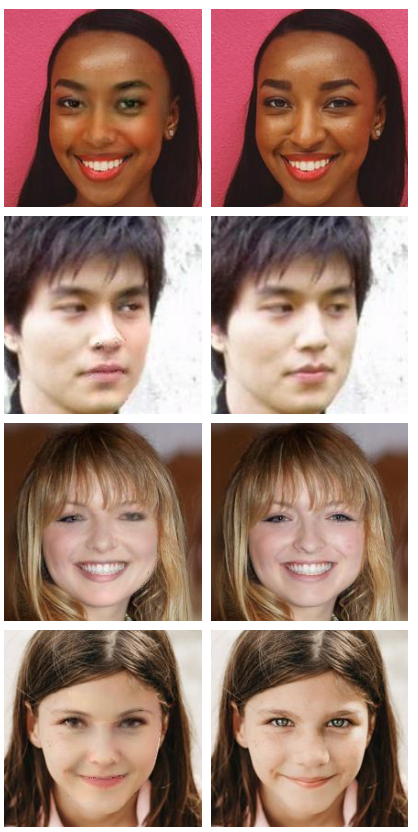

3DFaceFill (Ours)

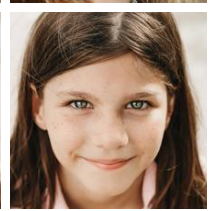

Ground Truth

Figure 5: Qualitative Evaluation: Inpainting on faces from the CelebA $\mid 24]$ and CelebA-HQ [18] test sets (except last row downloaded from the internet). Across a variety of scenarios, the completions from baselines often have artifacts while those from 3DFaceFill are significantly more photorealistic due to explicit modelling of the image formation process. More examples can be found in the supplementary.

Qualitative Evaluation: Fig.5 5 qualitatively compares face completion between 3DFaceFill and the baselines over a wide variety of challenging conditions. Completions by the baselines are less photorealistic and often contain artifacts in scenarios with dark complexion, tend to deform facial components (e.g. nose) and fail to preserve symmetry 

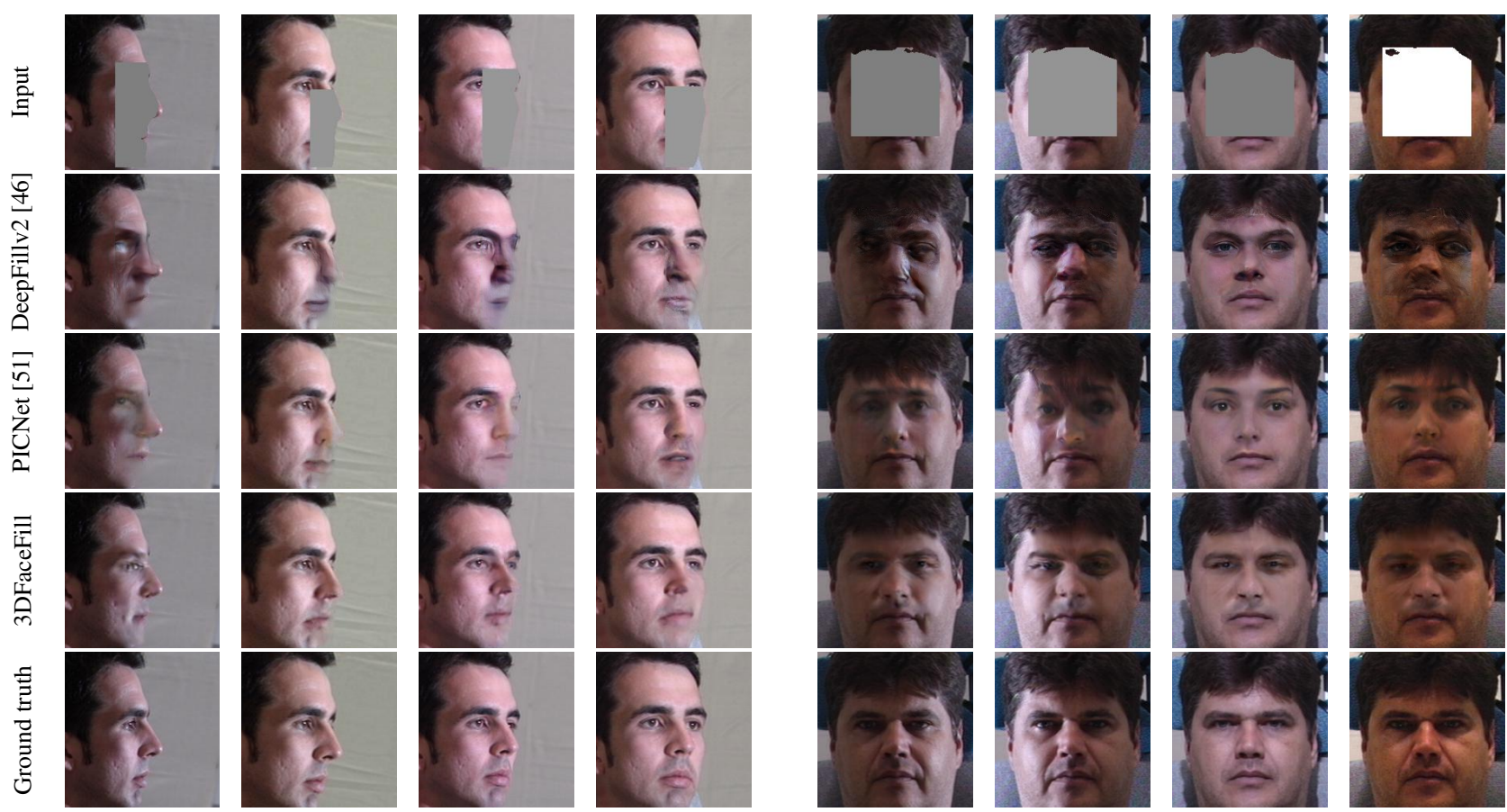

Figure 6: Qualitative evaluation on the MultiPIE [10] dataset. Compared to the baselines that generate deformed faces with artifacts in extreme poses and illumination, 3DFaceFill is more robust and generate geometrically accurate and illumination-preserving faces.

(e.g. eye-gaze or eye-brow shape). In addition, the baselines tend to deform the shape of small faces (e.g. children) since they are mostly trained on adult faces where the relative proportions of facial parts differs significantly. In contrast, 3DFaceFill generates more photorealistic completions in all these cases (diverse conditions and mask types) due to explicit 3D shape modeling, incorporating symmetry priors and disentanglement of pose and illumination.

Cross-Dataset Evaluation: To further demonstrate the improved generalization performance and robustness afforded by our method, we perform a cross-dataset comparison on the pose and illumination varying images from the MultiPIE [10] dataset, using models that were trained on the CelebA dataset [24]. Note that most baselines [20, 45, 51, 52] do not perform such an evaluation. Quantitative results are in the last rows of Table 4, while Fig. 6 shows the qualitative results. Fig 6 (left) shows that the baselines generate fuzzy and deformed faces while 3DFaceFill generates consistently superior completion across all poses. Similarly, for the varying illumination case (Fig 6 . right), 3DFaceFill not only generates superior completion but also preserves the illumination contrast across the face.

Real Occlusions: One of the potential applications of face completion is in de-occlusion. This is usually challenging when faces have large pose, illumination or shape variations. Fig. 7 shows a few real-world de-occlusion examples of faces in such conditions. Notice that, in cases of

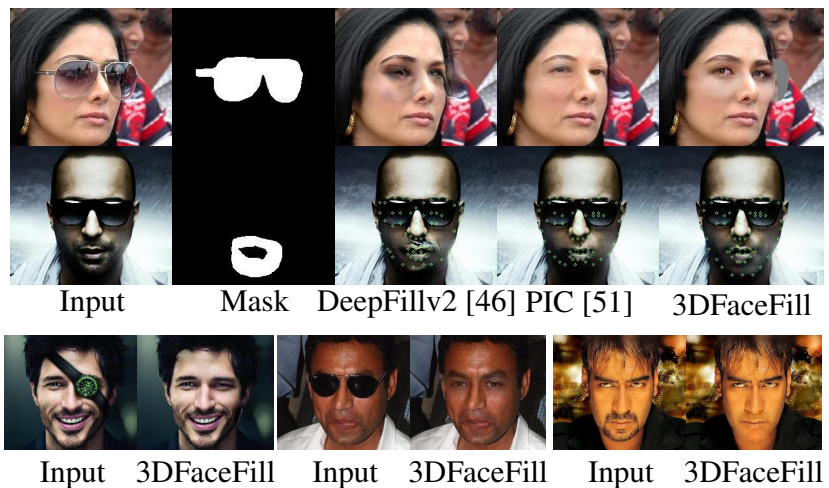

Figure 7: FC on real occlusions. Note the asymmetric eye-gaze (row 1) and blurry shape (row 2) by the baselines.

challenging pose, illumination, etc., the baselines tend to generate blurry and asymmetric face completions, whereas 3DFaceFill does more realistic de-occlusion.

\subsection{Ablation Studies}

Iterative Refinement: To evaluate the effectiveness of iteratively refining face completion at inference, we compare the PSNR, SSIM and LPIPS [49] metrics on raw output images (before blending with the visible image) at each iteration. As reported in Table 1, iteration 2 significantly improves upon iteration 1 over all the metrics. After iteration 2, the metrics become more or less stable, with a slight dip in performance. We hypothesize that it is a result of 


\begin{tabular}{ccccccc} 
& Iter 1 & Iter 2 & Iter 3 & Iter 4 & Iter 5 & Iter 6 \\
\hline PSNR $(\uparrow)$ & 33.7587 & $\mathbf{3 4 . 5 3 4 7}$ & 34.5018 & 34.4943 & 34.4428 & 34.4018 \\
SSIM $(\uparrow)$ & 0.9510 & $\mathbf{0 . 9 6 7 8}$ & 0.9675 & 0.9670 & 0.9666 & 0.9652 \\
LPIPS $(\downarrow)$ & 0.0192 & $\mathbf{0 . 0 1 8 5}$ & 0.0186 & 0.0187 & 0.0188 & 0.0188
\end{tabular}

Table 1: Quantitative evaluation of iterative refinement.

\begin{tabular}{cccccc}
\hline Metric & Full GAN & Patch GAN & NoSym & NoSym+Attn & Full Model \\
\hline PSNR $(\uparrow)$ & 31.7125 & 31.7552 & 31.6110 & 31.7969 & $\mathbf{3 2 . 1 9 5 0}$ \\
SSIM $(\uparrow)$ & 0.9654 & 0.9658 & 0.9665 & 0.9667 & $\mathbf{0 . 9 6 7 8}$ \\
LPIPS $(\downarrow)$ & 0.0462 & 0.0454 & 0.0446 & 0.0442 & $\mathbf{0 . 0 4 1 0}$ \\
\hline
\end{tabular}

Table 2: Quantitative evaluation between the different ablation models and our full model on masks blocking one-half of the face.

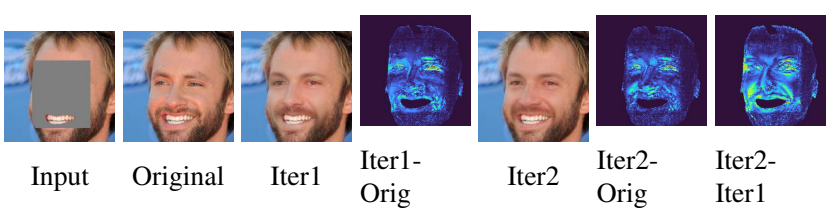

Figure 8: Visualization of raw completion (without blending) at iterations 1 and 2 along with the difference heatmaps.

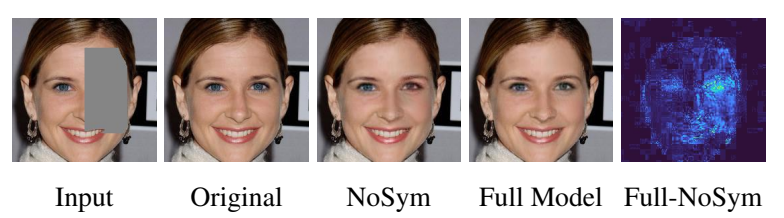

Figure 9: Visualizing the effect of symmetry for face inpainting. The full model includes Sym-UNet and symmetry loss (during training) and can copy symmetric features when available. The absolute difference heatmaps (Full-NoSym) shows that most difference is coming from components such as eyes, eye-brows, etc.

not training the model for iterative refinement and only performing it at inference. Further, we visualize the absolute difference heatmaps between the completed and the original image for both iterations 1 and 2 in Fig. 8 to understand which parts of the face benefit most from refinement. Observe that the largest differences are around the high-detail regions (eyes, beards, etc.), which we ascribe to more accurate $3 \mathrm{D}$ pose and shape estimation from the completed face after iteration 1 than from the partial face before.

Symmetry Constraint: To evaluate the effectiveness of Sym-UNet and the symmetry loss, we compare two variants of our full model. These include, (1) NoSym: Sym-UNet replaced by standard UNet and with no symmetry loss, and (2) NoSym+Attn: NoSym model plus a self-attention layer after the 3rd upsampling layer in the UNet decoder. Attention layers are commonly employed by many inpainting models [45, 46, 51] for capturing long-range spatial dependencies, so this variant seeks to compare the utility of attention in lieu of symmetry priors for face inpainting. To best evaluate the benefit of symmetry constraints for faces, the above model variations are evaluated on face images masked on one side of the face as shown in Fig. 9

The results in Table 2 indicate that the full model outper- forms all the variants, with NoSym being the worst among them. Also the NoSym+Attn variant does perform slightly better than NoSym but is still far behind the full model. This indicates that, (i) though attention helps in the absence of any prior constraints, explicitly enforcing geometric priors associated with structured objects like faces is significantly more effective than implicitly learning them through attention, and (ii) symmetry is a more useful feature for face inpainting and behaves like an attention on the visible symmetric parts. As shown in Fig. 9 compared to the full model, the NoSym variant results in larger inpainting errors as indicated by the difference heatmaps. Therefore, unlike the full model the NoSym model tends to ignore the visible symmetric regions of the face leading to inconsistencies between the visible and inpainted regions.

\subsection{Discussions}

The above described experiments and ablation studies demonstrate the effectiveness of 3DFaceFill, along with the utility of each of its components in performing robust face completion in challenging cases of facial pose, shape, illumination, etc. However, the formulation of our proposed approach do impose a dependency on the fidelity of the underlying 3D model. Essentially, our approach cannot inpaint on regions which are not included in the underlying 3D model and the resolution of inpainting depends on the density of the 3D mesh. 3DFaceFill currently uses the BFM model [28], thanks to its widespread support. However, BFM [28] does not include the inner mouth, hairs and the upper head and has limited vertex density around the eyes, which restricts inpainting in these regions. However, these limitations of the underlying 3D model are not inherent to the proposed approach and do not invalidate the advantages of our model in improving the geometric and photometric consistency of completion. Furthermore, these limitations can potentially be mitigated by substituting BFM with a more detailed 3D face model, such as the Universal Head Model (UHM) [29], that includes the inner mouth and detailed eye-balls, along with other improvements.

\section{Conclusion}

In this paper, we proposed 3DFaceFill, a 3D-aware face completion method. Our solution was driven by the hypothesis that performing face completion on the UV representation, as opposed to $2 \mathrm{D}$ pixel representation, will allow us to effectively leverage the power of 3D correspondence and ultimately lead to face completions that are geometrically and photometrically more accurate. Experimental evaluation across multiple datasets and against multiple baselines show that face completions from 3DFaceFill are significantly better, both qualitatively and quantitatively, under large variations in pose, illumination, shape and appearance. These results validate our primary hypothesis. 
Missing eye-brows

Blurred eyes and nose,
Illumination contrast

Blurry cheeks

Asymmetric eye-gaze

Blurry deformation near mouth and asymmetric eye-gaze

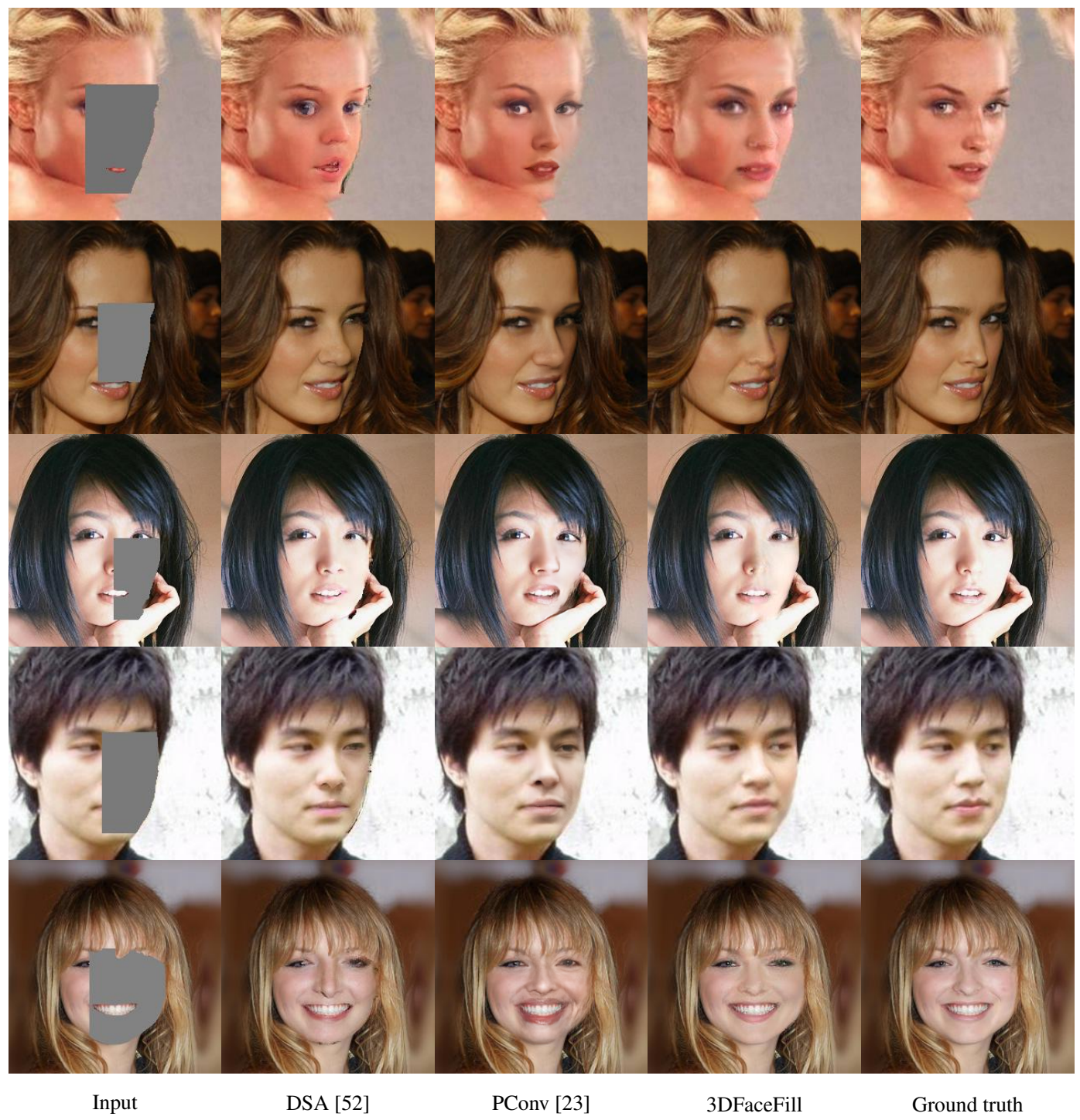

Figure 10: Qualitative evaluation of 3DFaceFill vs. PConv [23] and DSA [52] on a subset of images received from the respective authors. The text on the left mention the specific deformities in the baselines (blurriness, artifacts, asymmetry and other geometric deformations), that is not present in the completions by $3 \mathrm{DFaceFill}$.

\begin{tabular}{cccc}
\hline & PSNR $(\uparrow)$ & SSIM $(\uparrow)$ & LPIPS $[49](\downarrow)$ \\
\hline DSA [52] & 28.6205 & 0.9375 & 0.0436 \\
PConv $[\overline{23}]$ & 29.3067 & 0.9479 & 0.0379 \\
3DFaceFill & $\mathbf{3 1 . 8 8 2 3}$ & $\mathbf{0 . 9 6 1 5}$ & $\mathbf{0 . 0 3 3 5}$ \\
\hline
\end{tabular}

Table 3: Quantitative comparison of the proposed 3DFaceFill vs. PConv [23] and DSA [52] on a small set of completed images obtained from the authors.

\section{Appendices}

\section{A. Experimental Results}

\section{A.1. Comparison against PConv [23] and DSA [52]}

PConv [23] and DSA [52] have not released publicly available source codes or pre-trained models. Hence, to compare against them, we obtained face completions for a small set of 14 partial images through correspondence with the respective authors 3 We show qualitative results in Fig. 10. One can observe that while PConv [23] and DSA [52] tend to deform the facial components under certain conditions leading to geometric and photometric artifacts, 3DFaceFill is free of such artifacts and generates more realistic completions. In addition, we provide quantitative metrics on this small set in Table 3, where 3DFaceFill reports better PSNR, SSIM and LPIPS [49] metrics over both

\footnotetext{
${ }^{3}$ The images provided by PConv's authors were obtained from a model trained on $512 \times 512$ sized images, vs. $256 \times 256$ for the other baselines including 3DFaceFill.
} 


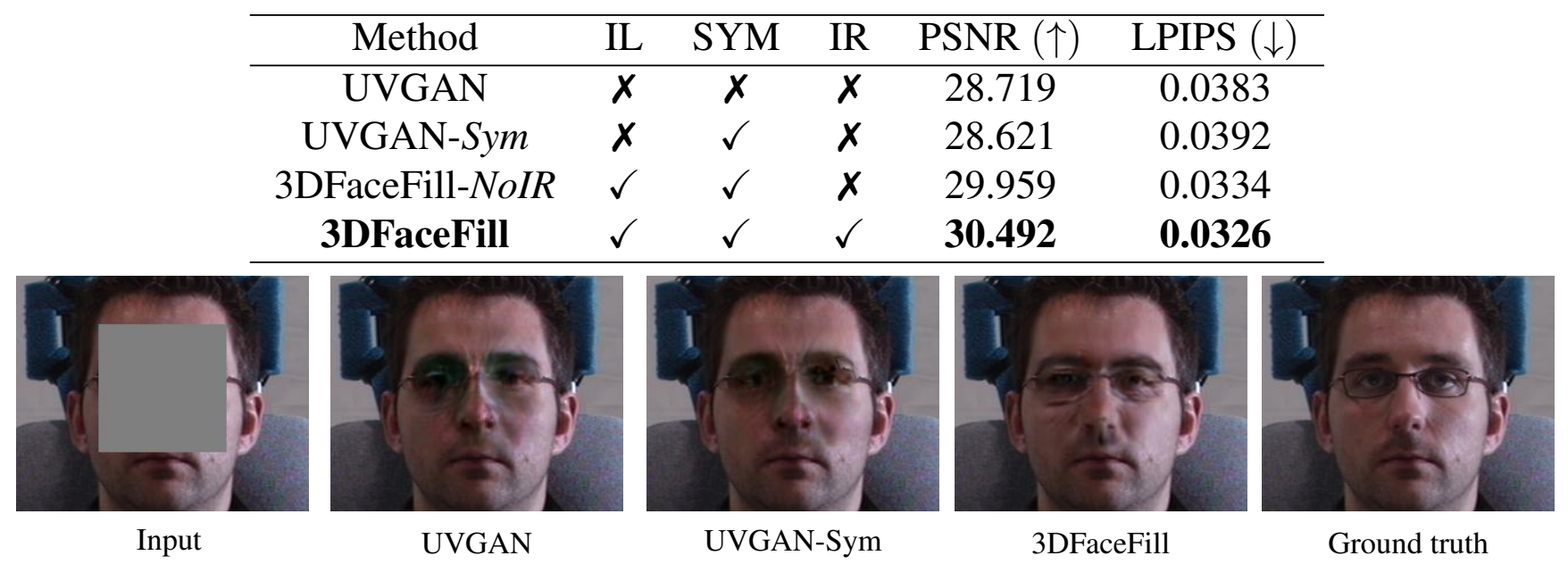

Figure 11: Comparing UVGAN [7] reformulated for face completion vs. 3DFaceFill.

the baselines.

\section{A.2. Comparison against UVGAN [7]}

The proposed face completion method, 3DFaceFill, has three parts, (i) disentangling 2D image into factors such as $3 \mathrm{D}$ pose, 3D shape, albedo and illumination (IL), (ii) enforcing symmetry in UV albedo $(S Y M)$, and (iii) iterative refinement of face completion through progressively more accurate $3 \mathrm{D}$ pose and shape estimation (IR). UVGAN [7] on the other hand, (i) performs completion of the missing texture in the UV-representation due to self-occlusion instead of completing a partial face image itself, (ii) unlike 3DFaceFill, does not disentangle texture further into albedo and illumination, (iii) does not impose symmetry prior on the UV texture, and (iv) uses 3DMM on a fully visible face image rather than a partial image to obtain texture. Since no source code or pretrained model of UVGAN is available, we evaluate these differences in two ways: (A) by reformulating UVGAN for face completion, and (B) comparing UVGAN with our Sym-UNet model on their publicly released texture dataset. We now present the two evaluations.

\section{A: Comparison with UVGAN [7] Reformulated for Face Completion}

To simulate UVGAN [7] for face completion, we remove the illumination disentanglement $(I L)$, symmetry loss $(S Y M)$ and iterative refinement (IR) from 3DFaceFill (refer to Fig. 11). We call the variant with $S Y M$ as UVGAN$S y m$, and the variant with both $I L$ and $S Y M$ as 3 DFaceFillNoIR. Adding IR makes for our full model 3DFaceFill. We compare the above-mentioned variants for face completion on the CelebA [24] dataset and report the quantitative and qualitative results in Fig. 11. One can observe that 3DFaceFill significantly outperforms UVGAN as well as the other variants both quantitatively as well as qualita- tively. Further, we can see that introducing the symmetry loss $(S Y M)$ in UVGAN-Sym hurts performance since, unlike UV-albedo, UV-texture is not inherently symmetric in faces because of the entangled illumination. Completion on the disentangled albedo $(I L)$ instead improves performance in 3DFaceFill-NoIR. Lastly, iterative refinement $(I R)$ further improves completion on top of $I L$ and SYM. This demonstrates the effectiveness of the novelties that 3DFaceFill introduces over UVGAN [7].

\section{B: Sym-UNet vs. UVGAN on Texture Completion}

In this evaluation, we trained our Sym-UNet model on the UVDB-MPIE texture dataset released by the authors of UVGAN [7]. We split the dataset into a 80:20 train-test split and resized the texture maps to $192 \times 256$ for training. Similar to UVGAN, we do not include the symmetry loss because of the presence of illumination variations and the availability of synthetically completed texture maps, which reduces the utility of symmetry-loss. The rest of the Sym-UNet is retained as such. On the test set, we report a PSNR of 30.1 (vs. UVGAN's 25.8) and SSIM of 0.937 ( $v s$. UVGAN's 0.886). Further, we show qualitative results in Fig. 12, where we see that our completed textures resemble the ground truth closely (we do not have the corresponding completions by UVGAN). Thus, our proposed Sym-UNet network is comparatively better suited for UV-completion than the network used in UVGAN [7].

\section{A.3. Further Qualitative Evaluation}

\section{A: Diverse Conditions}

We present further qualitative comparison of face completion by the proposed 3DFaceFill vs. DeepFillv2 [46] and PIC [51] under diverse conditions. Fig. 13 show the qualitative comparison on faces with dark complexion, challenging poses and illumination contrast. Fig. 14 shows 

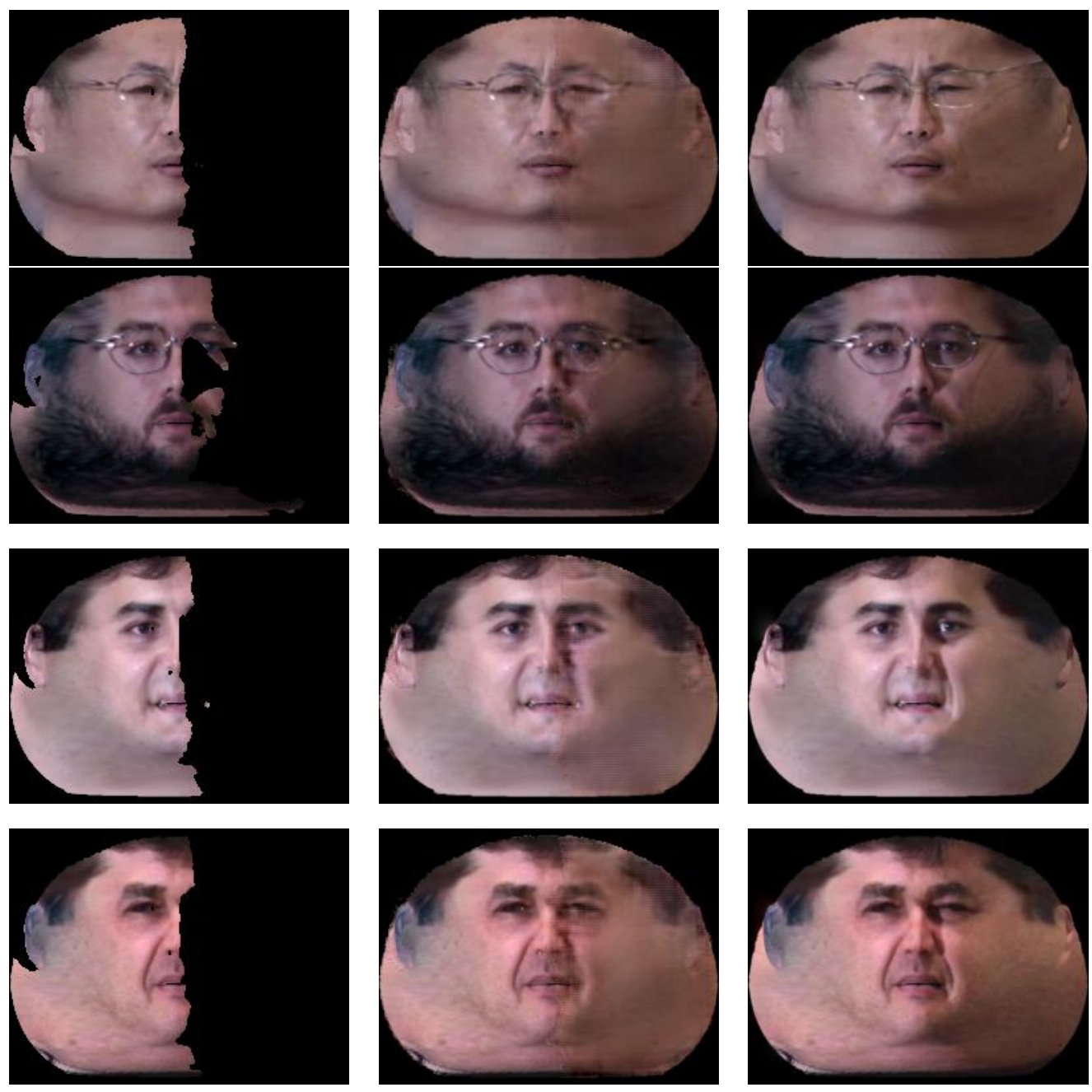

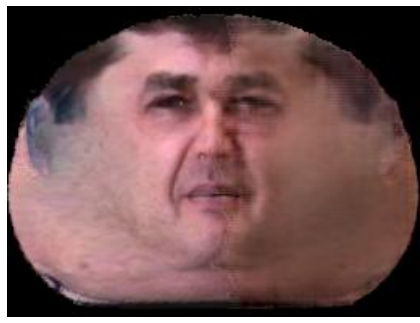

(b) 3DFaceFill

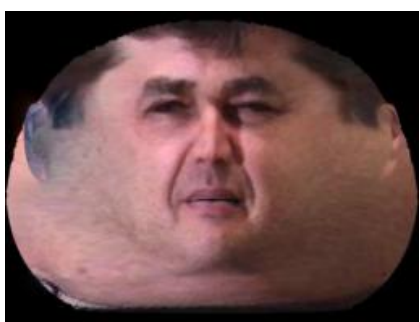

(c) Groundtruth

Figure 12: Qualitative evaluation of texture completion by the proposed Sym-UNet on the UVDB-MPIE dataset [7].

examples where baselines tend to introduce asymmetry in eye-gaze or deform various face components, such as, nose, mouth, etc. In all these cases, 3DFaceFill generates more realistic completions while preserving the visible illumination contrast and bilateral symmetry, because of the disentangled completion of albedo and explicit enforcement of $3 \mathrm{D}$ shape, pose, illumination and symmetric priors.

\section{B: Robustness Across Pose and Illumination Variations}

We present further cross-dataset evaluation on the pose and illumination varying MultiPIE dataset [10] by splitting the dataset into two subsets: (1) a pose varying subset with constant frontal illumination and expression, referred to as MultiPIE:Pose and (2) an illumination varying subset with constant frontal pose and expression, referred to as MultiPIE:Illu. Table 4 reports the PSNR, SSIM and LPIPS [49] metrics for all the methods on these two splits. It can be seen that 3DFaceFill significantly outperforms the baselines in both the splits. Further, we show more example completions by 3DFaceFill vs. the baselines DeepFillv2 [46] and PIC [51] in Fig. 15(for Pose) and Fig. 16 (for Illumination), respectively. From Fig. 15, one can observe that the baselines tend to generate fuzzy and deformed faces for extreme poses while 3DFaceFill generates sharper and geometrypreserving completions. And, in the illumination-varying case, DeepFillv2 [46] tends to generate artifacts and PIC [51] tends to generate asymmetric completions for extreme illumination, whereas the completions by 3DFaceFill are free of such artifacts and preserve illumination contrast and symmetry.

\section{C: Generalization Performance on In-the-Wild Images}




\section{DARKER COMPLEXION}
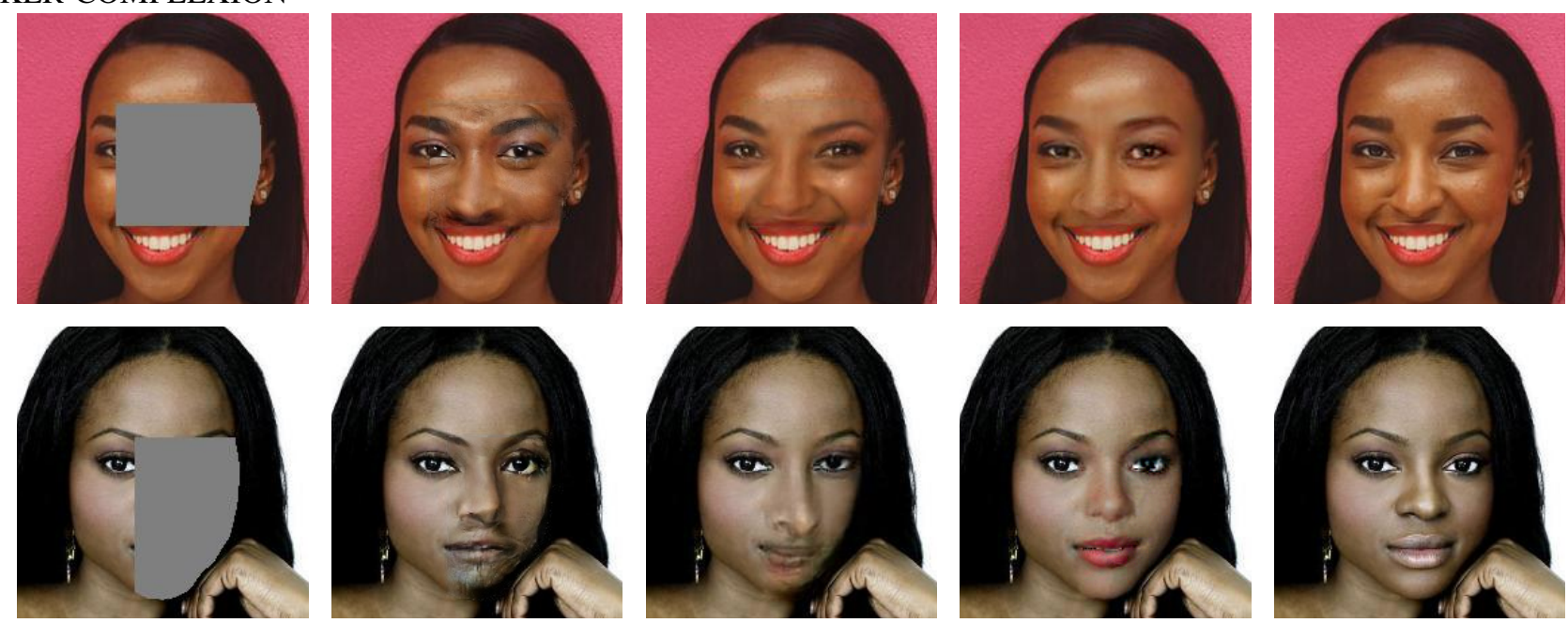

\section{LARGE POSES}
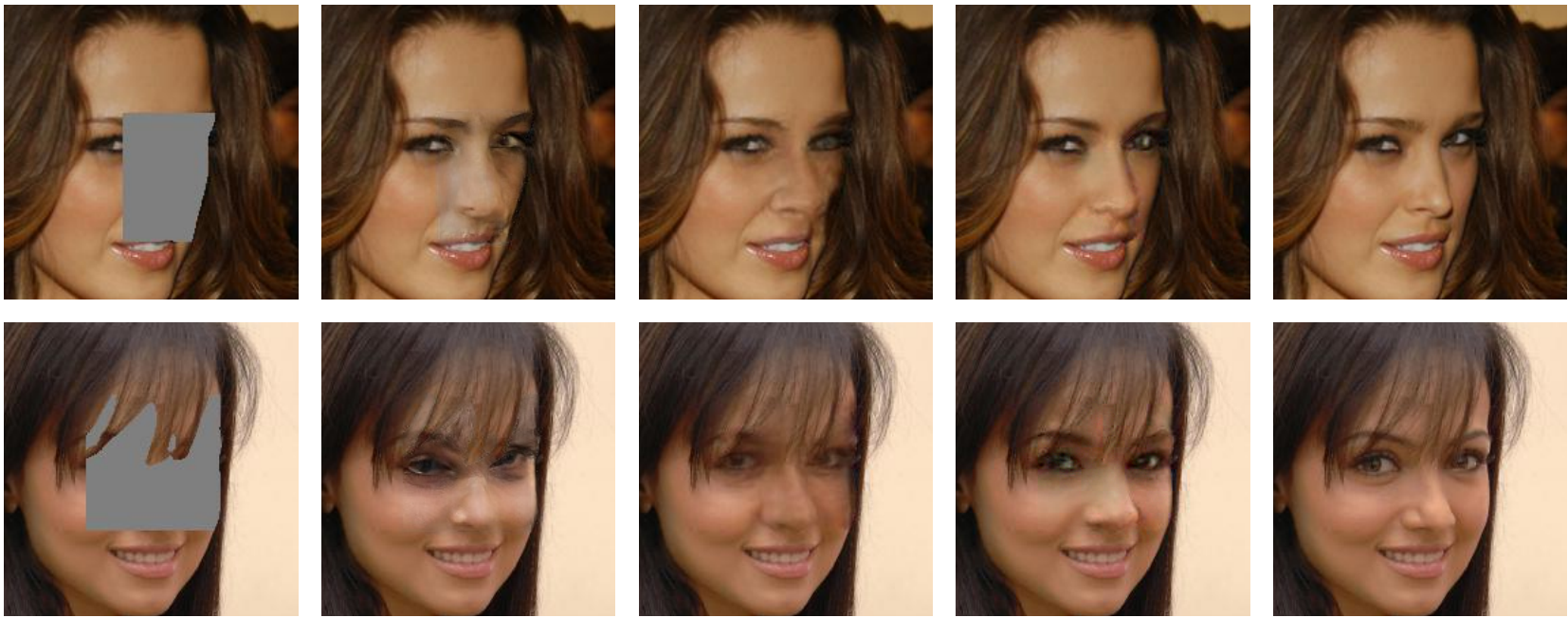

\section{ILLUMINATION CONTRAST}
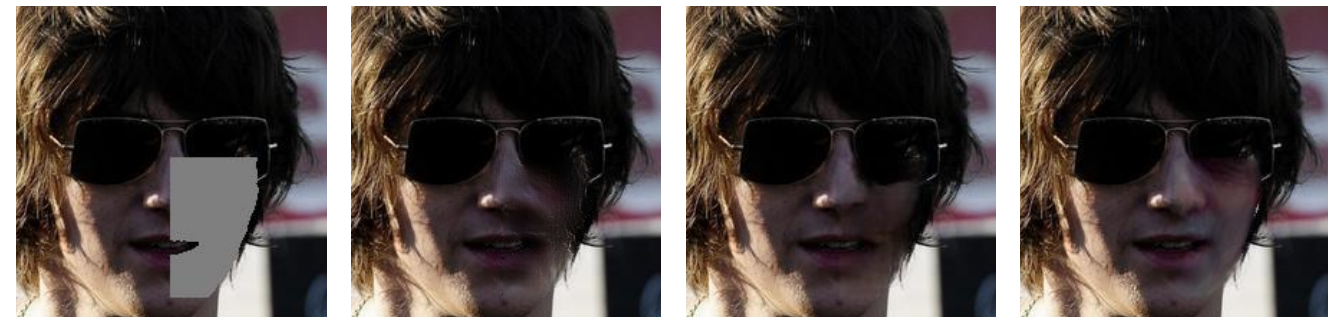

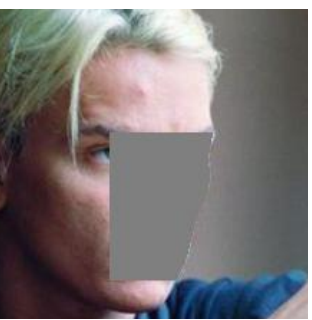

Input

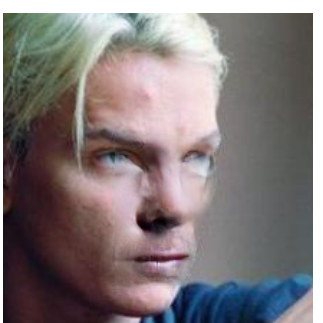

DeepFillv2 [46]

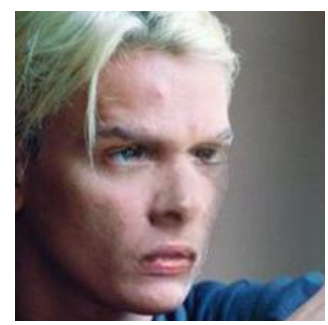

PIC [51]

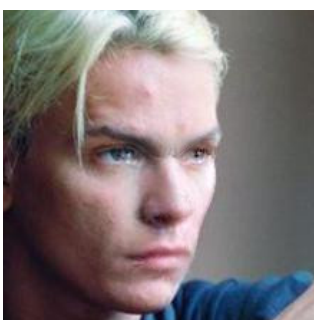

3DFaceFill (Ours)
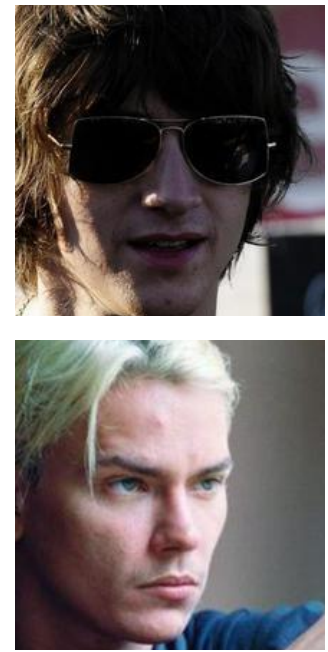

Ground Truth

Figure 13: Qualitative evaluation under diverse conditions (complexion, pose, illumination). 
ASYMMETRY IN EYE-GAZE
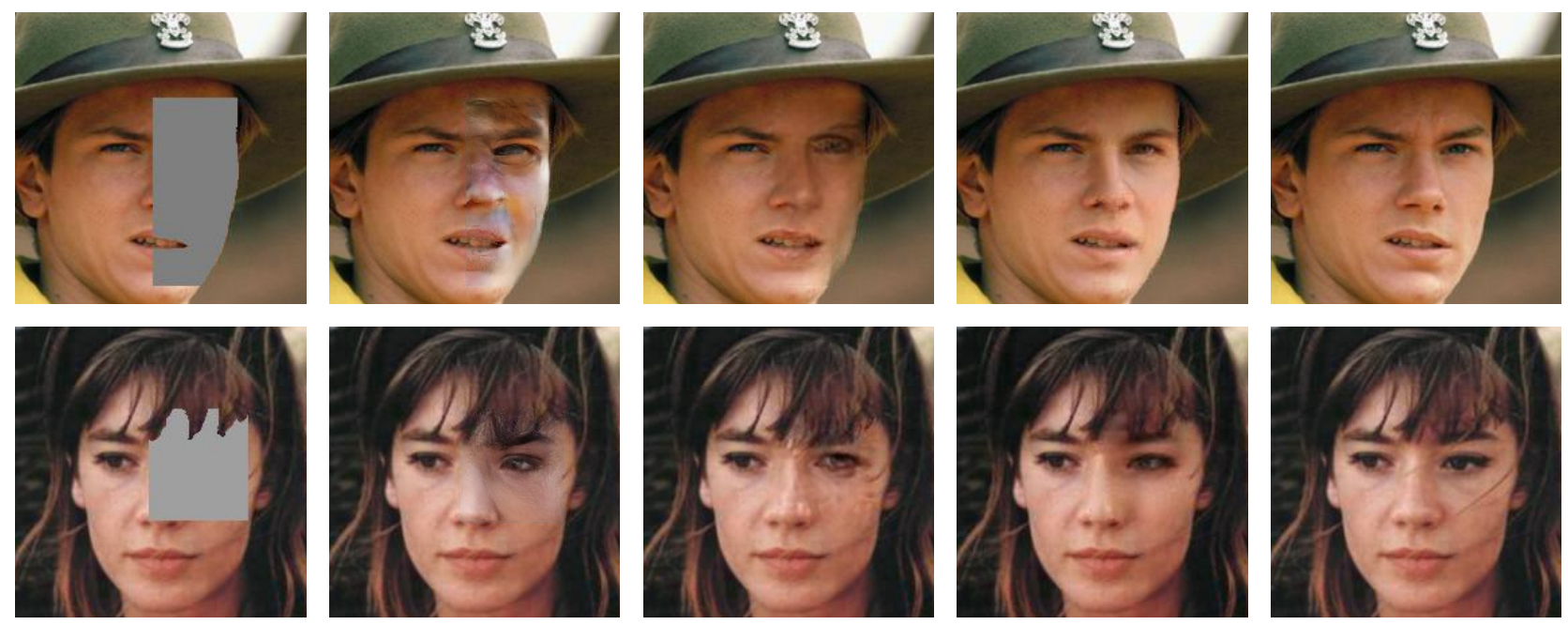

SHAPE DEFORMATIONS
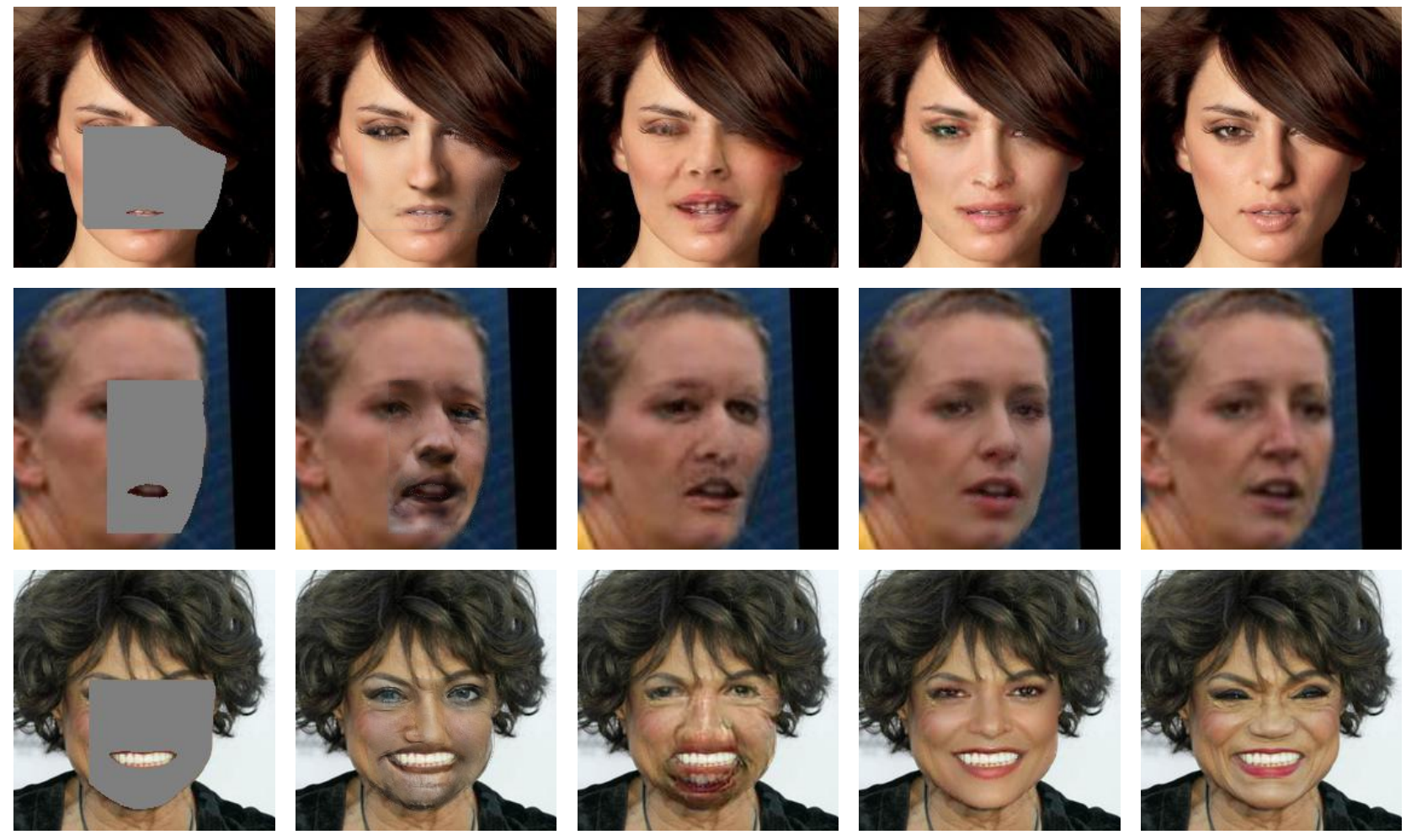

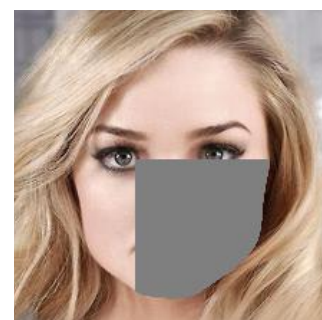

Input

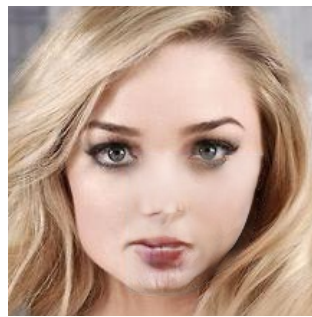

DeepFillv2 [46]

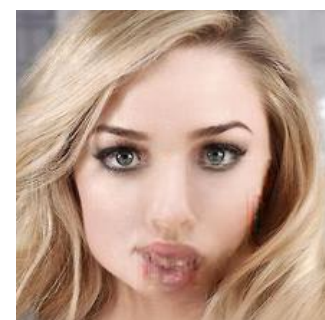

PIC [51]

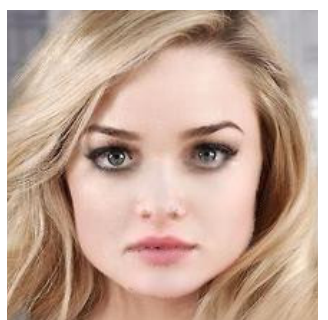

3DFaceFill (Ours)

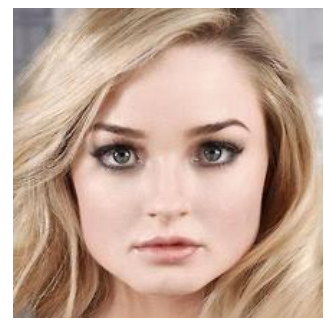

Ground Truth

Figure 14: Qualitative evaluation under diverse conditions (eye-gaze, shape). 

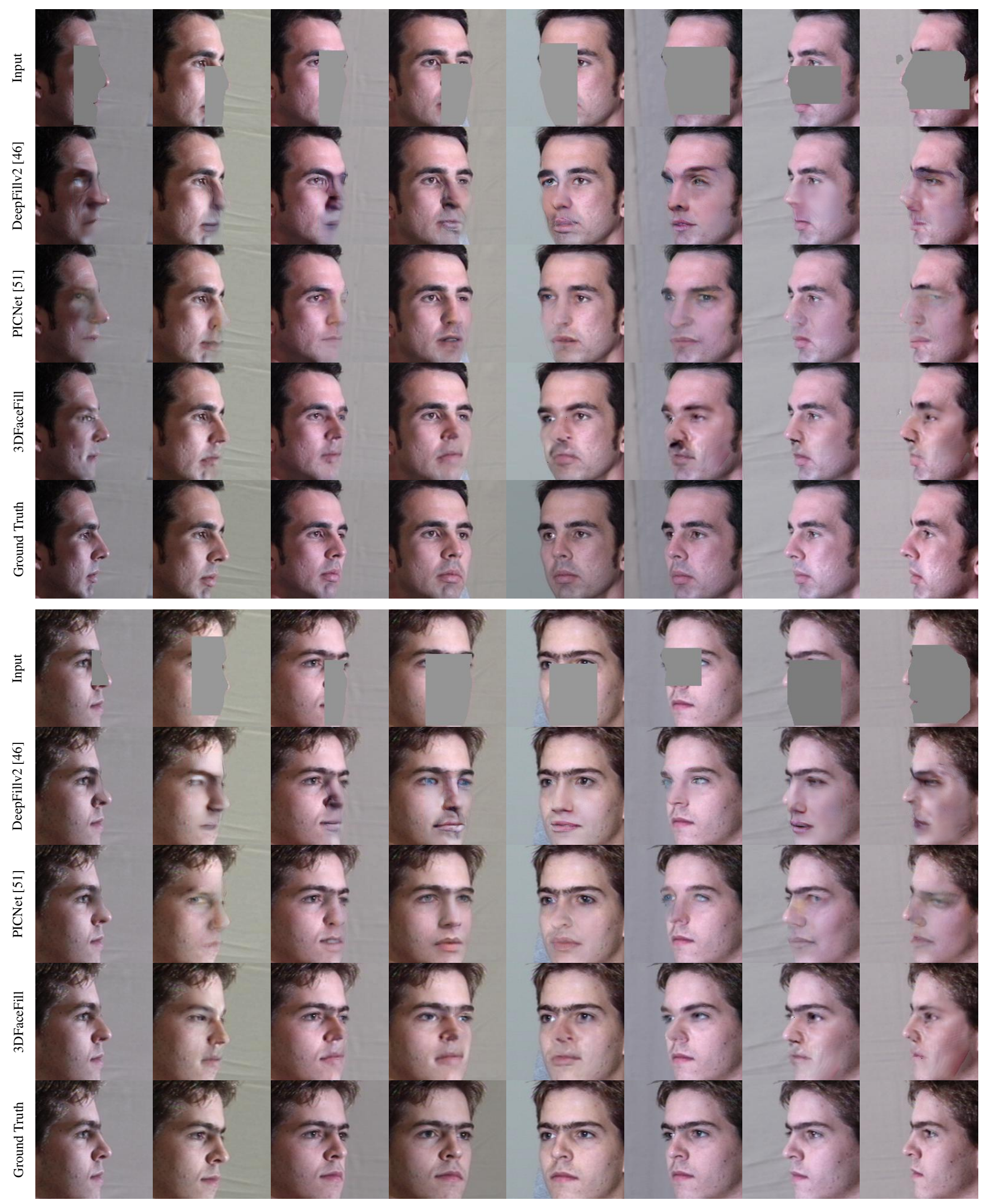

Figure 15: Qualitative evaluation of 3DFaceFill vs. baselines DeepFillv2 [46] and PIC [51] on the pose-varying MultiPIE:Pose split [10]. While the baselines tend to generate blurred and deformed faces in extreme poses, 3DFaceFill is pose-robust and generates more accurate completions across a range of pose. 

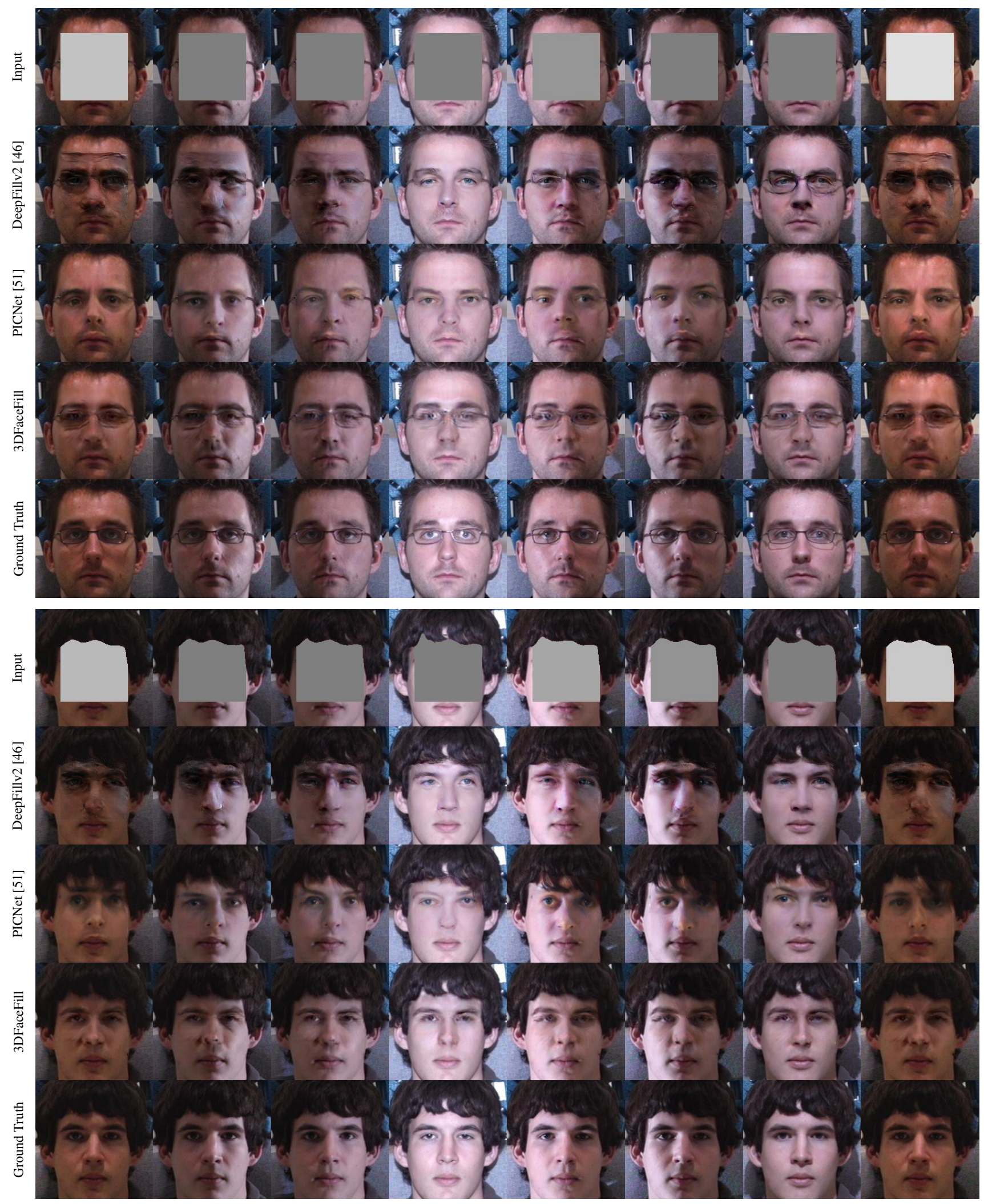

Figure 16: Qualitative evaluation of 3DFaceFill vs. the baselines DeepFillv2 [46] and PIC [51] on the illumination varying MultiPIE:Illu split [10]. While the baselines tend to generate artifacts in extreme illuminations, 3DFaceFill generates completions that look geometrically accurate and preserve the illumination contrast (notice (i) the illumination contrast in cols. 2,3,5,6 (b), and (ii) assymetric eye-brows in cols. 1,2,3,5,6 (b) by the baselines.) 


\begin{tabular}{llccccc}
\hline Dataset & Metric & GFC $[\overline{20}]$ & SymmFC $[19]$ & DeepFillv2 $[46]$ & PIC $[51]$ & 3DFaceFill \\
\hline \multirow{3}{*}{ MultiPIE:Pose } & PSNR $(\uparrow)$ & 24.7557 & 24.7177 & 26.3385 & 26.4301 & $\mathbf{2 7 . 8 2 2 6}$ \\
& SSIM $(\uparrow)$ & 0.9187 & 0.9289 & 0.9383 & 0.9451 & $\mathbf{0 . 9 4 8 2}$ \\
& LPIPS $(\downarrow)$ & 0.0822 & 0.0692 & 0.0527 & 0.0471 & $\mathbf{0 . 0 4 0 9}$ \\
\hline \multirow{3}{*}{ MultiPIE:Illu } & PSNR $(\uparrow)$ & 23.5749 & 24.4813 & 26.4981 & 26.2938 & $\mathbf{2 7 . 8 8 6 5}$ \\
& SSIM $(\uparrow)$ & 0.8676 & 0.8618 & 00.8718 & 0.8825 & $\mathbf{0 . 8 9 3 5}$ \\
& LPIPS $(\downarrow)$ & 0.1232 & 0.0747 & 0.0640 & 0.0540 & $\mathbf{0 . 0 4 8 4}$ \\
\hline \multirow{3}{*}{ Internet } & PSNR $(\uparrow)$ & 24.1775 & 24.2829 & 26.4957 & 25.6326 & $\mathbf{2 8 . 8 4 6 3}$ \\
& SSIM $(\uparrow)$ & 0.9042 & 0.9168 & 0.9293 & 0.9317 & $\mathbf{0 . 9 5 2 6}$ \\
& LPIPS $(\downarrow)$ & 0.0913 & 0.0625 & 0.0493 & 0.0466 & $\mathbf{0 . 0 3 9 0}$ \\
\hline
\end{tabular}

Table 4: Further quantitative evaluation of 3DFaceFill vs. the baselines on the pose-varying (MultiPIE:Pose) and illumination varying (MultiPIE:Illu) subsets of the MultiPIE dataset [10] and in-the-wild images downloaded from the Internet.
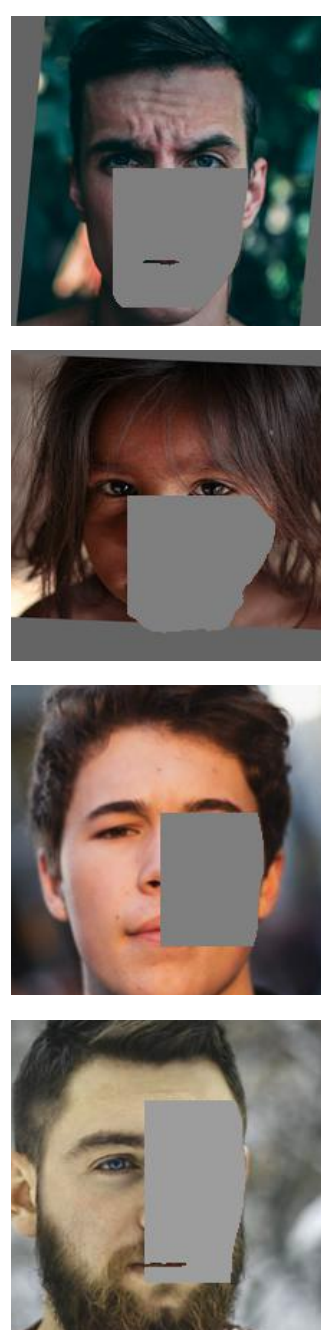

Input
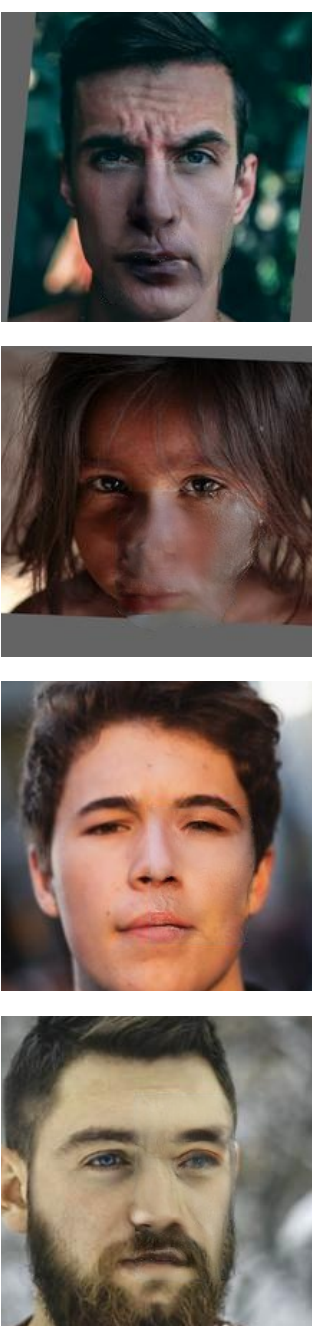

DeepFillv2 [46]
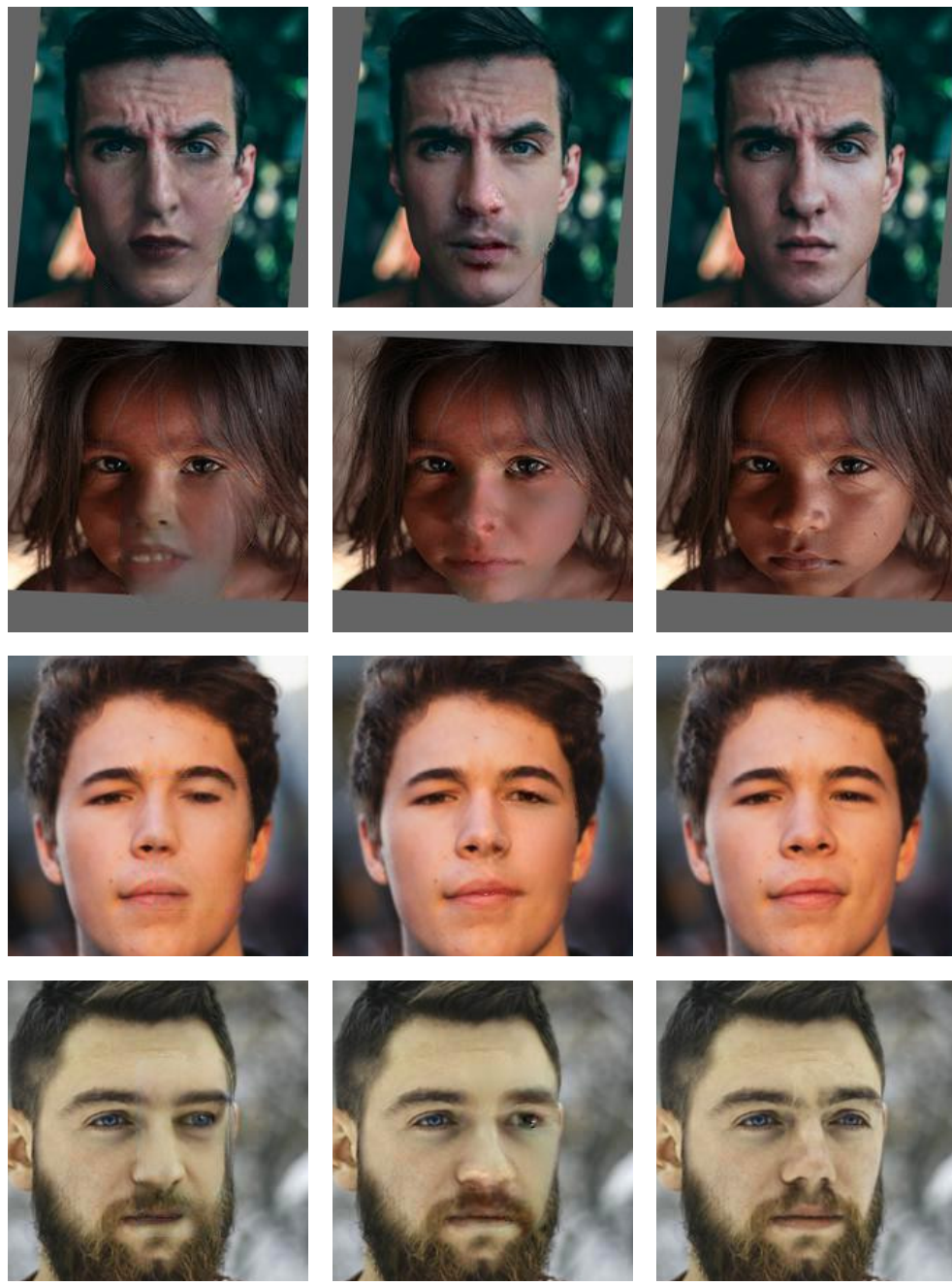

PIC [51]

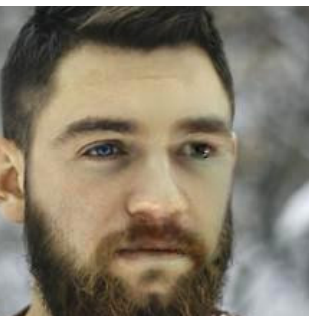

3DFaceFill (Ours)
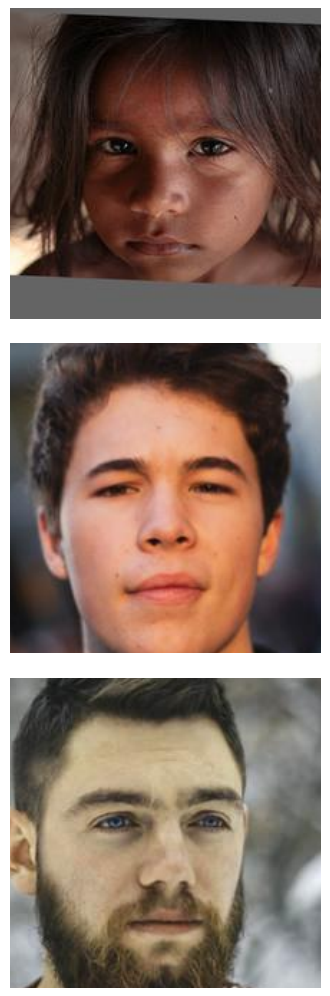

Ground Truth

Figure 17: Qualitative evaluation (of generalization performance) on the Internet downloaded images. 


\section{downloaded from the Internet}

To compare the generalization performance of different methods, we evaluate face completion on a small dataset of $\sim 50$ in-the-wild face images downloaded from the interne ${ }^{4}$ (referred to as Internet). We report the quantitative metrics in Table 4, where one can see significant margins between 3DFaceFill and the closest baselines across all the three metrics, demonstrating the better generalization performance of our proposed method. Fig. 17 shows qualitative comparison on a small sample where 3DFaceFill generates more realistic completions, thanks to the explicit imposition of 3D face priors. This shows that the principles behind 3DFaceFill can improve the generalization performance of image completion approaches on structured objects such as faces.

\section{A.4. 3D View Synthesis of Masked Faces}

3DFaceFill has a unique advantage over other face completion approaches, in that unlike existing methods, our method can not only complete partial faces, but also render new views of the completed face from different view-points. In Fig. 18, we show this through examples of face views rendered from five different viewpoints by completing the missing albedo and self-occluded regions in the masked faces.

\section{A.5. Evaluation in terms of SSIM}

In addition to the PSNR/LPIPS vs. mask ratio analysis reported in the main paper, we also report similar comparison in terms of SSIM vs. mask ratio on the CelebA [24], CelebA-HQ [18] and MultiPIE [10] datasets in Fig. 19a. Fig. 19b and Fig. 19c, respectively. 3DFaceFill consistently out-performs the baselines in terms of SSIM too for all the mask ratios. Moreover, it can be observed that the comparative gain by 3DFaceFill vs. the closest baseline increases as the mask ratio increases, which we attribute to the advantage of using explicit 3D priors for completion.

\section{B. Analysis}

\section{B.1. Iterative Refinement}

As explained in Main:Sec 3.2, we adopt an iterative refinement procedure whereby $3 \mathrm{D}$ factorization aids in completion and vice versa. We show heatmap-visualization of the difference between the pre-blended completions at iterations 1 and 2 in Fig. 20. A relative comparison of the distribution of Iter1-GT and Iter2-GT shows that Iter2 is closer to the GT (ground truth) image, which indicates improved 3D pose estimation in the second iteration. The visualization of Iter2-Iter1 shows that these differences are manifesting at the detailed face components such as eyes, nose, etc., as well as the masked regions.

\footnotetext{
${ }^{4}$ Source: https://unsplash.com/s/photos/face
}

\section{B.2. Effect of Symmetry}

We further analyze the advantages of enforcing symmetry-consistency. We show completions by the Full model (Sym-UNet + symmetry loss) and the NoSym model (UNet) in Fig. 21 The completions by the NoSym variant look slightly asymmetric because of blurry and symmetryagnostic completion around the eyes and other masked regions whereas our Full model leverages symmetry to generate sharper and symmetry-consistent completions. This can be further visualized in the Full-NoSym difference heatmaps shown in the figure that show that the difference is mostly concentrated around the eyes and eye-brows and spreads further through the masked regions.

Symmetry Gating Activation: We further visualize the intermediate gating maps used in our model that control the flow of information in the network (ref Fig. 22). We visualize two (out of 64) gating activations (1st - Gate1 and 33rd - Gate2) from the second layer of our Sym-UNet network. As can be seen in Fig. 22, while Gate1 activates for the visible regions in the input albedo, Gate 2 activates for the masked regions to propagate useful features from the horizontally flipped albedo map to the symmetric side. This enables Sym-UNet to leverage and maintain facial symmetry for inpainting. We also visualize the estimated uncertainty map $(\sigma)$ in Fig. 22 that is learned by the inpainter $\mathcal{G}$ in an unsupervised way. Note that the uncertainty is usually higher around important facial components like the eyes and the masked regions, which increases the loss incurred in these regions.

\section{Implementation Details}

In this section, we provide further implementation details regarding the proposed approach. In sub-section C.1. we give detailed network architectures for the modules used in 3DFaceFill. In sub-section C.2, we provide details of the loss functions used to train the $3 \mathrm{D}$ factorization module. Lastly, we give full training details of the different components in sub-section C.3

\section{C.1. Network Architectures}

We report the detailed network architectures for the 3 DMM Encoder $\mathcal{E}$, the Albedo Decoder $\mathcal{D}_{A}$, the Sym-UNet module, the PyramidGAN discriminator and the Face Segmenter $\mathcal{S}$ in Tables 5 to 9 Our network architectures for the 3DMM modules are based on the architectures used in [38] for the corresponding modules. Insipired by Miyato et al. [26], we use spectral normalization in all our convolution layers. The abbreviated operators used are defined as follows:

- $\operatorname{Conv}\left(c_{i n}, c_{\text {out }}, k, s, p\right): 2 \mathrm{D}$ convolution with $c_{i n}$ input channels, $c_{\text {out }}$ output channels, kernel size $k$, stride $s$ and padding $p$. 

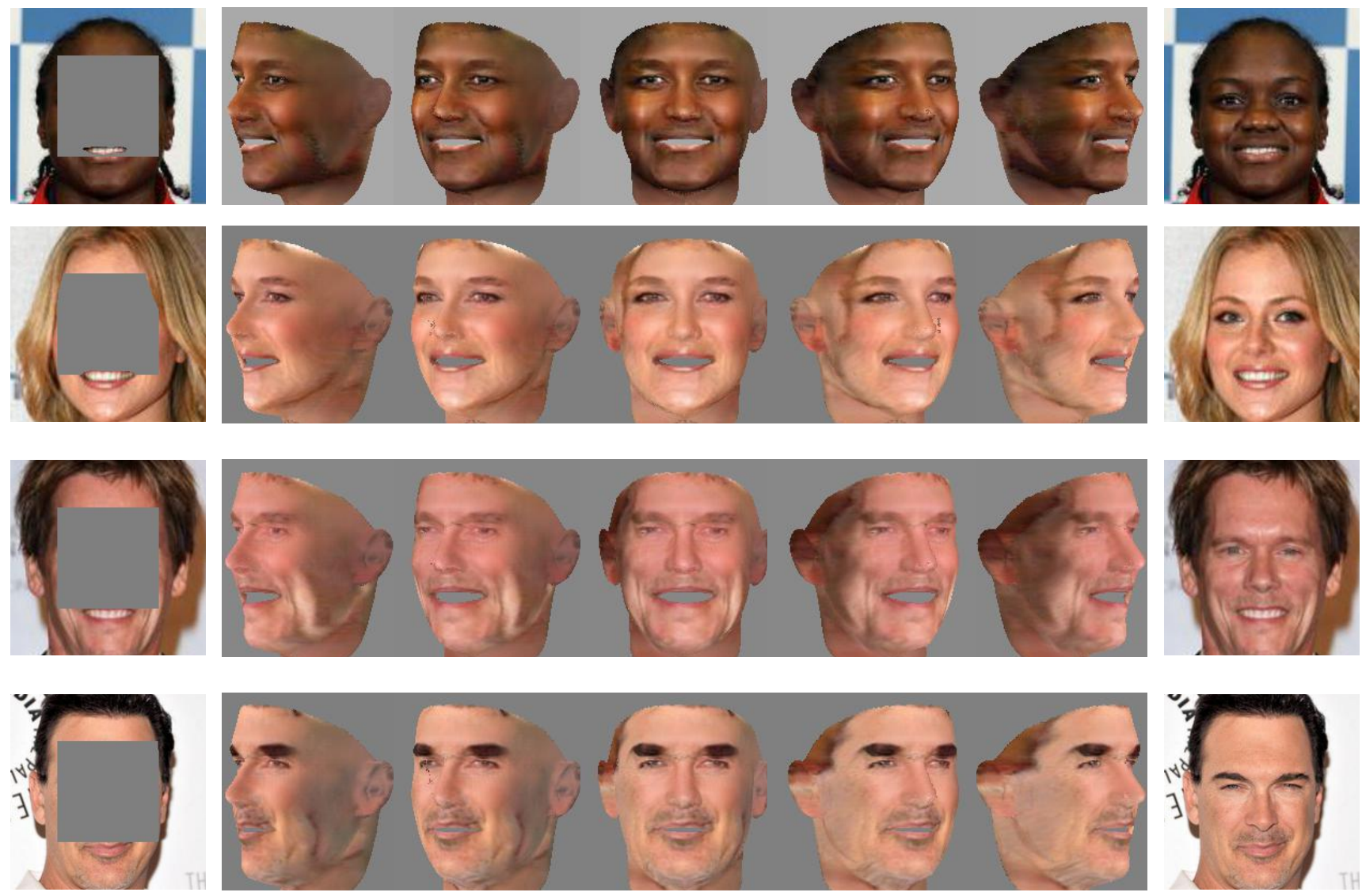

(a) Input

(b) Completed and synthesized face views

(c) Ground Truth

Figure 18: 3D Face View Synthesis. 3DFaceFill has the unique ability to not just complete masked faces realistically, but also synthesize new views from them.

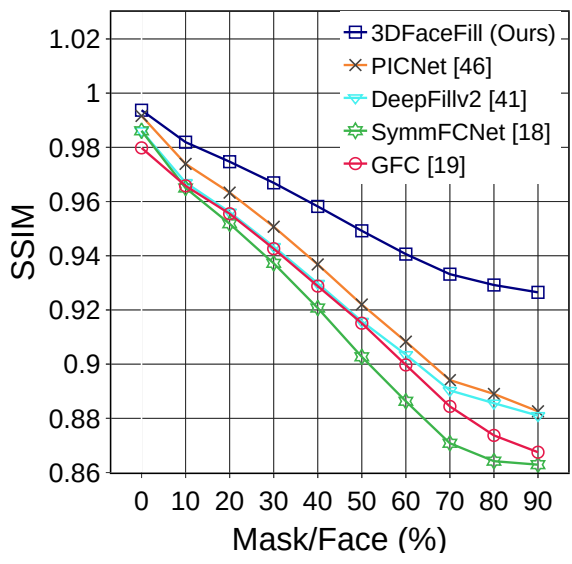

(a) CelebA dataset 24

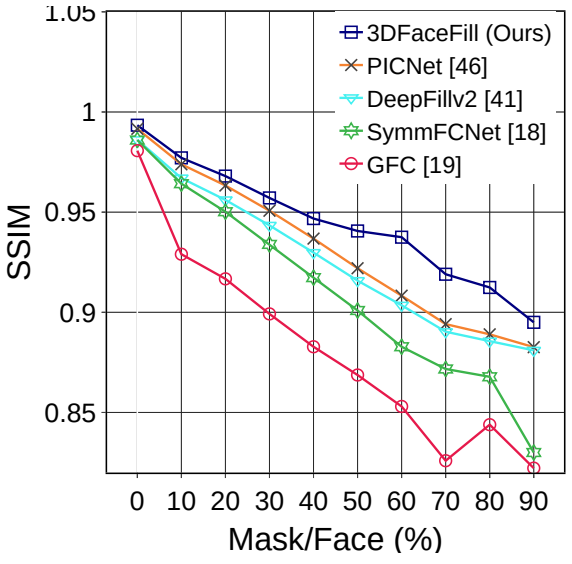

(b) CelebA-HQ dataset $\mid 18$

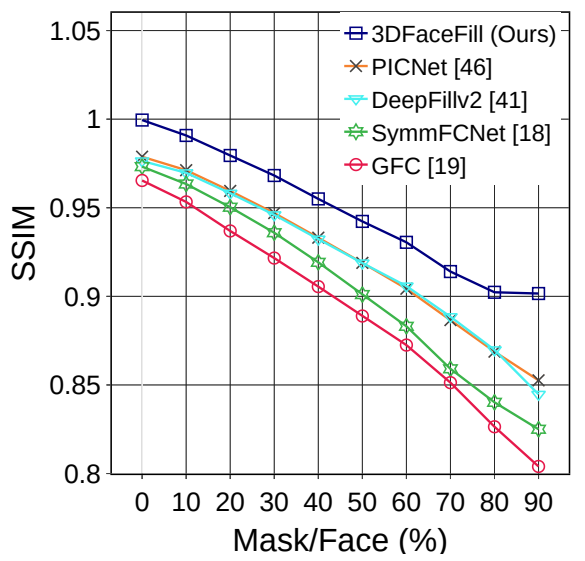

(c) MultiPIE dataset $[10$

Figure 19: Quantitative evaluation of 3DFaceFill $v s$. the baselines in terms of SSIM. 3DFaceFill consistently and significantly outperforms the baselines across all the Mask-to-Face area ratios and across all the datasets viz. CelebA [24], CelebA-HQ [18] and MultiPIE [10].

- $\operatorname{Deconv}\left(c_{i n}, c_{o u t}, k, s, p\right): 2 \mathrm{D}$ transposed convolution (deconvolution) with $c_{i n}$ input channels, $c_{o u t}$ output channels, kernel size $k$, stride $s$ and padding $p$. 


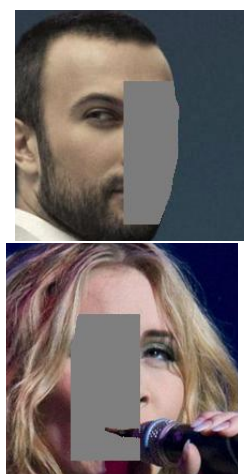

Input

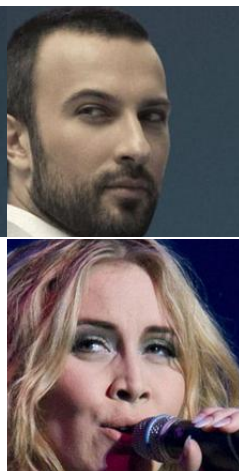

Ground Truth

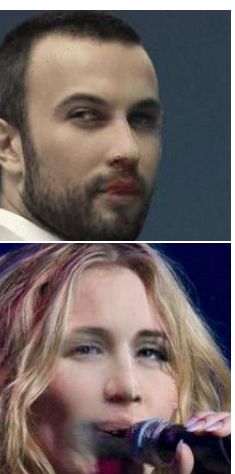

Iter1

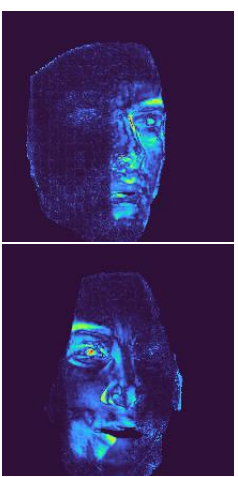

Iter1 - GT

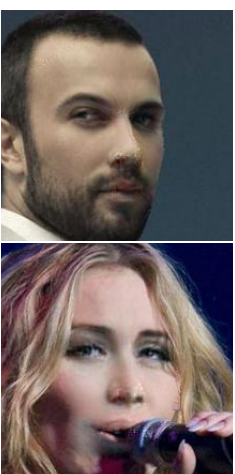

Iter2

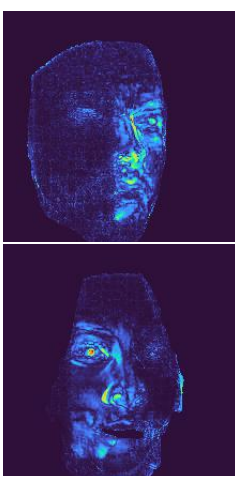

Iter2 - GT

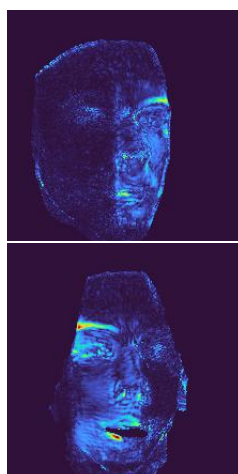

Iter2 - Iter1

Figure 20: Effect of Iterative Finetuning. We show raw completions (without blending) at iterations 1 and 2 along with the difference heatmaps. Note the improvements in Iter 2 over Iterl and the corresponding heatmap activations around eyes, eye-brows and other edges on the face.
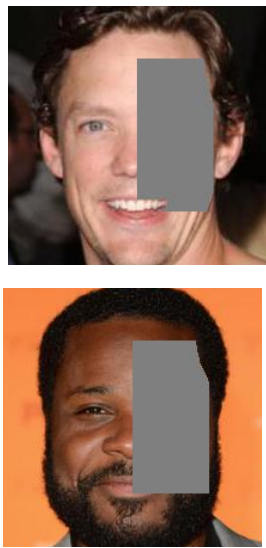

Input
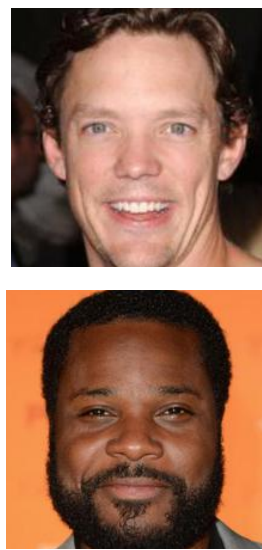

Ground Truth
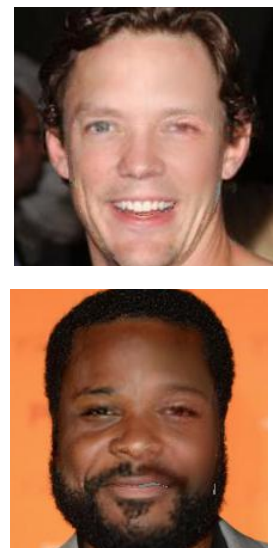

NoSym Model
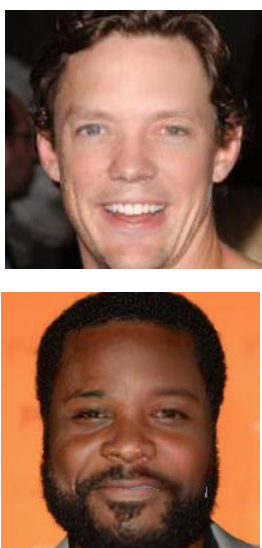

Full Model
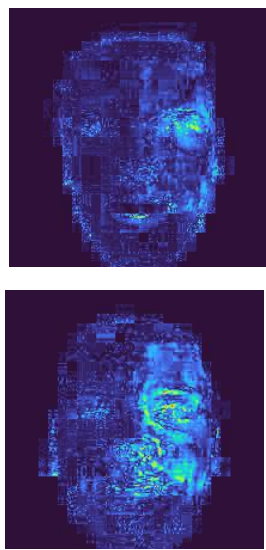

Full-NoSym

Figure 21: Effect of using Symmetry. The full model includes Sym-UNet and symmetry loss (during training) and can copy symmetric features when available. The absolute difference heatmaps (Full-NoSym) shows that most difference is coming from components such as eyes, eye-brows, etc.

- $\mathrm{GN}(n)$ : Group normalization [43] with $n$ groups

- ELU: Exponential linear unit [5] activation, $\operatorname{LReLU}(\alpha)$ : Leaky ReLU [25] with a negative slope of $\alpha$

- ResUnit $\left(c_{i n}, c_{o u t}, k, s, p\right)$ : Residual unit [13] with $c_{i n}$ input channels, $c_{\text {out }}$ output channels, kernel size $k$, stride $s$, padding $p$ with group normalization [43] and ELU activation [25]

- $\operatorname{SigGNConv}\left(c_{i n}, c_{o u t}, k, s, p\right)$ : 2D convolution with $c_{i n}$ input channels, $c_{\text {out }}$ output channels, kernel size $k$, stride $s$ and padding $p$ followed by group normalization [43] and sigmoid activation

- $\operatorname{SigGNDeconv}\left(c_{i n}, c_{o u t}, k, s, p\right):$ 2D transposed convolution with $c_{i n}$ input channels, $c_{\text {out }}$ output channels, kernel size $k$, stride $s$ and padding $p$ followed by group normalization [43] and sigmoid activation
- SpectralConv $\left(c_{i n}, c_{o u t}, k, s, p\right):$ 2D convolution with $c_{\text {in }}$ input channels, $c_{\text {out }}$ output channels, kernel size $k$, stride $s$, padding $p$ and spectral normalization [26]

- Upsample $\left(s_{h}, s_{c}\right)$ : Upsamples height by $s_{h}$ and width by $s_{w}$ using nearest neighbour interpolation.

\section{C.2. 3DMM Module Losses}

The 3DMM module is trained using a combination of supervised, reconstruction and regularization losses:

$$
\mathcal{L}_{3 D M M}=\lambda_{\text {sup }} \mathcal{L}_{\text {sup }}+\lambda_{\text {rec }} \mathcal{L}_{\text {rec }}+\lambda_{\text {reg }} \mathcal{L}_{\text {reg }}
$$

where, $\quad \mathcal{L}_{\text {sup }}=\lambda_{S} \mathcal{L}(\mathbf{S}, \tilde{\mathbf{S}})+\lambda_{p} \mathcal{L}(\mathbf{p}, \tilde{\mathbf{p}})+$ $\lambda_{T} \mathcal{L}\left(\mathbf{T}^{u v}, \tilde{\mathbf{T}}^{u v}\right)+\lambda_{\text {lmark }} \mathcal{L}_{\text {lmark }}$ use the groundtruth shape, pose, texture and 2D landmarks when available, $\mathcal{L}_{\text {rec }}$ enforces similarity between the rendered and grountruth images and $\mathcal{L}_{\text {reg }}=\lambda_{3 \text { dsym }} \mathcal{L}_{3 d \text { sym }}+\lambda_{\text {const }} \mathcal{L}_{\text {const }}$ 

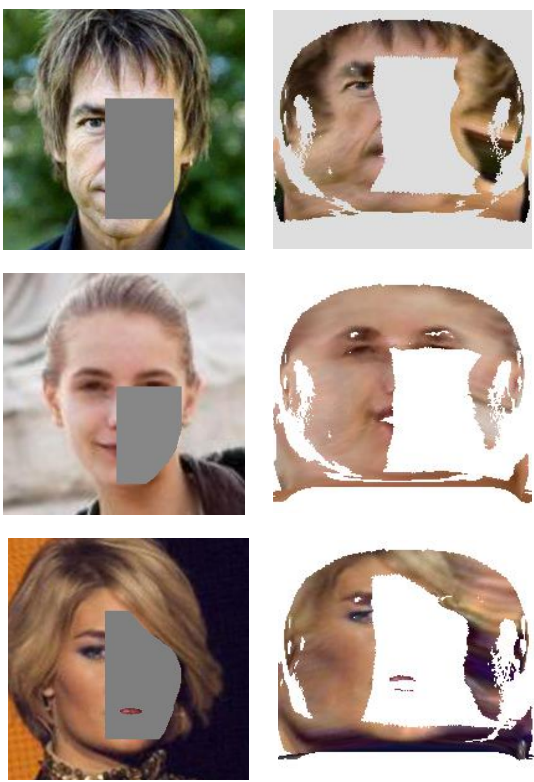

(a) Input
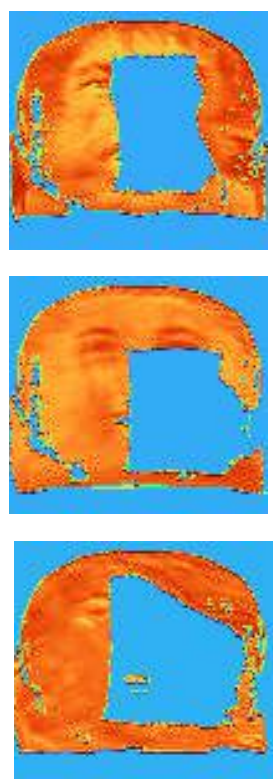

(c) Gate 1
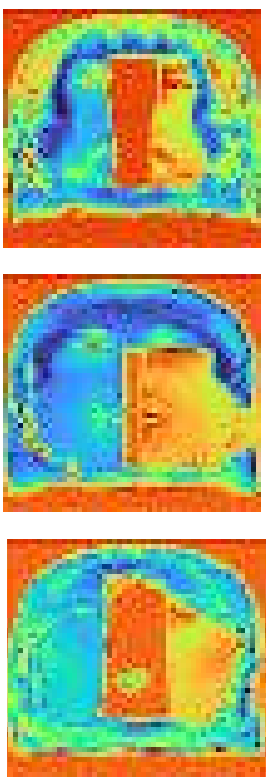

(d) Gate 2
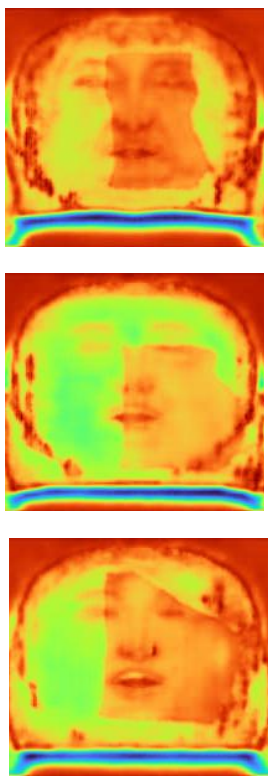

(e) Uncertainty $\sigma$
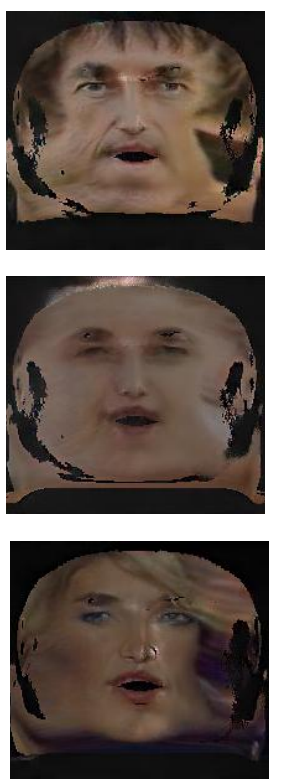

(f) Output Albedo

Figure 22: Visualizing the Gating Activations and the Uncertainty-Maps. Observe that, while Gate 1 activates for the visible regions, Gate 2 activates for the masked regions to propagate useful features from the visible symmetric parts to their masked counterparts. The uncertainty map captures the model's uncertainty around the masked regions and the facial components such as the eyes, thus incurring higher losses for these regions. (Note: higher values are represented by warmer (redish) colors in the gating and uncertainty heatmaps).

Table 5: Network architecture of the 3DMM Encoder $\mathcal{E}$. The Pose $_{1}$ corresponds to the scale, Pose $_{2: 4}$ correspond to the yaw, roll and pitch angles normalized by $\pi / 2$ and Pose $_{5: 6}$ correspond to the $\mathrm{X}$ and $\mathrm{Y}$ translations normalized by the input image size.

\begin{tabular}{|c|c|}
\hline 3DMM Encoder & Output size \\
\hline Image $\rightarrow$ SpectralConv $(3,32,7,2,3)+\mathrm{GN}(8)+\mathrm{ELU}$ & $112 \times 112$ \\
\hline SpectralConv $(32,64,3,1,1)+\mathrm{GN}(16)+\mathrm{ELU}$ & $112 \times 112$ \\
\hline SpectralConv $(64,64,3,2,1)+\mathrm{GN}(16)+\mathrm{ELU}$ & $56 \times 56$ \\
\hline SpectralConv $(64,96,3,1,1)+\mathrm{GN}(24)+$ ELU & $56 \times 56$ \\
\hline SpectralConv $(96,128,3,1,1)+\mathrm{GN}(32)+\mathrm{ELU}$ & $56 \times 56$ \\
\hline SpectralConv $(128,128,3,2,1)+\mathrm{GN}(32)+$ ELU & $28 \times 28$ \\
\hline SpectralConv $(128,196,3,1,1)+\mathrm{GN}(48)+\mathrm{ELU}$ & $28 \times 28$ \\
\hline SpectralConv $(196,256,3,1,1)+\mathrm{GN}(64)+$ ELU & $28 \times 28$ \\
\hline SpectralConv $(256,256,3,2,1)+\mathrm{GN}(64)+\mathrm{ELU}$ & $14 \times 14$ \\
\hline SpectralConv $(256,256,3,1,1)+\mathrm{GN}(64)+\mathrm{ELU}$ & $14 \times 14$ \\
\hline SpectralConv $(256,256,3,1,1)+\mathrm{GN}(64)+\mathrm{ELU}$ & $14 \times 14$ \\
\hline SpectralConv $(256,512,3,2,1)+\mathrm{GN}(128)+\mathrm{ELU}$ & $7 \times 7$ \\
\hline SpectralConv $(512,512,3,1,1)+\mathrm{GN}(128)+\mathrm{ELU} \rightarrow$ feats & $7 \times 7$ \\
\hline feats $\rightarrow$ SpectralConv $(512,160,3,1,1)+\mathrm{GN}(40)+\mathrm{ELU}$ & $7 \times 7$ \\
\hline $\operatorname{AvgPool}(7,7)$ & $1 \times 1$ \\
\hline \multicolumn{2}{|l|}{ Linear $(160,6)+$ Tanh $\rightarrow$ Pose } \\
\hline feats $\rightarrow$ SpectralConv $(512,160,3,1,1)+\mathrm{GN}(40)+$ ELU & $7 \times 7$ \\
\hline $\operatorname{AvgPool}(7,7)$ & $1 \times 1$ \\
\hline \multicolumn{2}{|l|}{ Linear $(160,27) \rightarrow$ Illumination } \\
\hline feats $\rightarrow$ SpectralConv $(512,512,3,1,1)+\mathrm{GN}(128)+\mathrm{ELU}$ & $7 \times 7$ \\
\hline $\operatorname{SpectralConv}(512,512,3,1,1)+\mathrm{GN}(128)+\mathrm{ELU}$ & $7 \times 7$ \\
\hline $\operatorname{AvgPool}(7,7)$ & $1 \mathrm{x} 1$ \\
\hline \multicolumn{2}{|l|}{ Linear $(512,199+29) \rightarrow 199$ Shape +29 Expression coefficients } \\
\hline feats $\rightarrow$ SpectralConv $(512,512,3,1,1)+\mathrm{GN}(128)+$ ELU & $7 \times 7$ \\
\hline AvgPool $(7,7) \rightarrow$ Albedo features & $1 \times 1$ \\
\hline Model Complexity & $17.4 \mathrm{M}$ \\
\hline
\end{tabular}

are regularization losses to enforce bilateral symmetry of albedo and effective separation of shade and albedo. All loss coefficients $\lambda$ 's are set to have equal weightage for all the loss terms. We now define these losses:

- Shape loss is defined as:

$$
\mathcal{L}(\mathbf{S}, \tilde{\mathbf{S}})=\mathbb{E}\left[\left\|\mathbf{f}_{S}-\tilde{\mathbf{f}}_{S}\right\|_{2}^{2}\right]
$$

where $\mathbf{f}_{S}$ and $\tilde{\mathbf{f}}_{S}$ are the output and groundtruth shape and expression coefficients, respectively.

- Pose loss is defined as a combination of scale, translation and rotation losses:

$\mathcal{L}(\mathbf{p}, \tilde{\mathbf{p}})=\lambda_{s} \mathbb{E}\left[(s-\tilde{s})^{2}\right]+\lambda_{t} \mathbb{E}\left[\left\|\mathbf{t}_{x, y}-\tilde{\mathbf{t}}_{x, y}\right\|_{2}^{2}\right]+\lambda_{r} \mathcal{L}_{R}$, where $s$ represents scale, $\mathbf{t}_{x, y}$ represents the $\mathrm{X}$ and $\mathrm{Y}$ translations, and $\mathcal{L}_{R}=\mathbb{E}\left[\|\operatorname{quat}(\mathbf{R})-\operatorname{quat}(\tilde{\mathbf{R}})\|_{2}^{2}\right]$ is the rotation loss with $\mathbf{R}$ representing the rotation along the $\mathrm{X}, \mathrm{Y}$ and $\mathrm{Z}$ axes and quat(.) gives its quaternion representation.

- Texture loss is defined as:

$$
\mathcal{L}\left(\mathbf{T}^{u v}, \tilde{\mathbf{T}}^{u v}\right)=\mathbb{E}\left[\left\|\mathbf{T}^{u v}-\tilde{\mathbf{T}}^{u v}\right\|_{2}^{2}\right]
$$

where $\mathbf{T}^{u v}$ is the texture represented in UV space. 
Table 6: Network architecture of the Albedo Decoder $\mathcal{D}_{A}$ that decodes the 512 dimensional Albedo features from the 3DMM Encoder $\mathcal{E}$ into $3 \times 192 \times 256$ dimensional Albedo representation in the UV space.

\begin{tabular}{|c|c|}
\hline Albedo Decoder & Output size \\
\hline Albedo features $\rightarrow$ Upsample $(3,4)$ & $3 \times 4$ \\
\hline SpectralConv $(512,512,3,1,1)+\mathrm{GN}(128)+\operatorname{ELU}$ & $3 \times 4$ \\
\hline SpectralConv $(512,256,3,1,1)+\mathrm{GN}(64)+\mathrm{ELU}$ & $3 \times 4$ \\
\hline Upsample $(2,2)$ & $6 \times 8$ \\
\hline SpectralConv $(256,256,3,1,1)+\mathrm{GN}(64)+\mathrm{ELU}$ & $6 \times 8$ \\
\hline SpectralConv $(256,128,3,1,1)+\mathrm{GN}(32)+\mathrm{ELU}$ & $6 \times 8$ \\
\hline SpectralConv $(128,128,3,1,1)+\mathrm{GN}(32)+\mathrm{ELU}$ & $6 \times 8$ \\
\hline Upsample(2,2) & $12 \times 16$ \\
\hline SpectralConv $(128,160,3,1,1)+\mathrm{GN}(40)+\mathrm{ELU}$ & $12 \times 16$ \\
\hline SpectralConv $(160,96,3,1,1)+\mathrm{GN}(32)+$ ELU & $12 \times 16$ \\
\hline SpectralConv $(96,128,3,1,1)+\mathrm{GN}(32)+\mathrm{ELU}$ & $12 \times 16$ \\
\hline Upsample $(2,2)$ & $24 \times 32$ \\
\hline SpectralConv $(128,128,3,1,1)+\mathrm{GN}(32)+\mathrm{ELU}$ & $24 \times 32$ \\
\hline SpectralConv $(128,64,3,1,1)+\mathrm{GN}(16)+$ ELU & $24 \times 32$ \\
\hline SpectralConv $(64,96,3,1,1)+\mathrm{GN}(24)+$ ELU & $24 \times 32$ \\
\hline Upsample $(2,2)$ & $48 \times 64$ \\
\hline SpectralConv $(96,96,3,1,1)+\mathrm{GN}(32)+\operatorname{ELU}$ & $48 \times 64$ \\
\hline SpectralConv $(96,64,3,1,1)+\mathrm{GN}(16)+\mathrm{ELU}$ & $48 \times 64$ \\
\hline SpectralConv $(64,64,3,1,1)+\operatorname{GN}(16)+$ ELU & $48 \times 64$ \\
\hline Upsample(2,2) & $96 \times 128$ \\
\hline SpectralConv $(64,64,3,1,1)+\mathrm{GN}(16)+\mathrm{ELU}$ & $96 \times 128$ \\
\hline SpectralConv $(64,32,3,1,1)+\mathrm{GN}(8)+\mathrm{ELU}$ & $96 \times 128$ \\
\hline SpectralConv $(32,32,3,1,1)+\mathrm{GN}(8)+\mathrm{ELU}$ & $96 \times 128$ \\
\hline Upsample $(2,2)$ & $192 \times 256$ \\
\hline SpectralConv $(32,32,3,1,1)+\mathrm{GN}(8)+\operatorname{ELU}$ & $192 \times 256$ \\
\hline SpectralConv $(32,16,3,1,1)+\operatorname{GN}(4)+\operatorname{ELU}$ & $192 \times 256$ \\
\hline SpectralConv $(16,16,3,1,1)+\operatorname{GN}(4)+\operatorname{ELU}$ & $192 \times 256$ \\
\hline \multicolumn{2}{|l|}{ Conv $(16,3,1,1,0)+$ Tanh $\rightarrow$ Albedo } \\
\hline Model Complexity & $5.54 \mathrm{M}$ \\
\hline
\end{tabular}

- Landmark loss is defined as:

$$
\mathcal{L}_{\text {lmark }}=\left\|\mathbf{M}(\mathbf{p}) *\left[\begin{array}{c}
\mathbf{S}(:, \mathbf{d}) \\
\mathbf{1}
\end{array}\right]-\mathbf{U}\right\|_{2}^{2},
$$

where $\mathbf{M}$ is the camera projection matrix obtained from the pose $\mathbf{p}, \mathbf{d}$ selects 68 indices corresponding to sparse 2D landmarks on the $3 \mathrm{D}$ face mesh $\mathbf{S}$ and $\mathbf{U} \in \mathbb{R}^{2 \times 68}$ are the groundtruth locations of 2D facial landmarks.

- Reconstruction is defined as:

$$
\mathcal{L}_{\text {rec }}=\mathbb{E}\left[\|\mathbf{I}-\hat{\mathbf{I}}\|_{1}\right]
$$

where $\mathbf{I}$ and $\tilde{\mathbf{I}}$ are the rendered and ground-truth images, respectively.

- Albedo symmetry loss is defined as:

$$
L_{3 d s y m}(\mathbf{A})=\left\|\mathbf{A}^{u v}-h f l i p\left(\mathbf{A}^{u v}\right)\right\|_{1},
$$

where $\mathbf{A}^{u v}$ is the UV representation of albedo and hflip() is the horizontal image flipping operation.
Table 7: Network architecture of the Albedo Inpainter $\mathcal{G}$ (SymUNet). The input to the network is the concatenation of the masked Albedo $\mathbf{A}_{m}^{u v}$ and the mask $\mathbf{M}^{u v}$ in the UV space $X=$

\begin{tabular}{|c|c|c|}
\hline Input & Layer & Output \\
\hline$X$ & $\operatorname{ResUnit}(4,32,3,2,1)$ & $f 1$ \\
\hline$X$ & $\operatorname{SigGNConv}(4,32,3,2,1)$ & $g 1$ \\
\hline$h f l i p(X)$ & $\operatorname{Res} U n i t(4,32,3,2,1)$ & $f 1^{\prime}$ \\
\hline$h \operatorname{flip}(X)$ & $\operatorname{SigGNConv}(4,32,3,2,1)$ & $g 1^{\prime}$ \\
\hline$\left(f 1 \odot g 1, f 1^{\prime} \odot g 1^{\prime}\right)$ & $\operatorname{ResUnit}(64,64,3,2,1)$ & $f 2$ \\
\hline$\left(f 1 \odot g 1, f 1^{\prime} \odot g 1^{\prime}\right)$ & $\operatorname{SigGNConv}(64,64,3,2,1)$ & $g 2$ \\
\hline$f 2 \odot g 2$ & $\operatorname{ResUnit}(64,128,3,2,1)$ & $f 3$ \\
\hline$f 2 \odot g 2$ & $\operatorname{SigGNConv}(64,128,3,2,1)$ & $g 3$ \\
\hline$f 3 \odot g 3$ & ResUnit(128, 256, 3, 2, 1) & $f 4$ \\
\hline$f 3 \odot g 3$ & $\operatorname{SigGNConv}(128,256,3,2,1)$ & $g 4$ \\
\hline$f 4 \odot g 4$ & $\operatorname{ResUnit}(256,512,3,2,1)$ & f5 \\
\hline$f 4 \odot g 4$ & $\operatorname{SigGNConv}(256,512,3,2,1)$ & $g 5$ \\
\hline$f 5 \odot g 5$ & $\operatorname{ResUnit}(512,256,3,1,1)$ & $f 5^{1}$ \\
\hline$f 5 \odot g 5$ & $\operatorname{SigGNConv}(512,256,3,1,1)$ & $g 5^{1}$ \\
\hline$f 5^{1} \odot g 5^{1}$ & Upsample(2,2) & $x 4$ \\
\hline$(x 4, f 4 \odot g 4)$ & $\operatorname{ResUnit}(512,128,3,1,1)$ & $f 4^{1}$ \\
\hline$f 5^{1} \odot g 5^{1}$ & $\operatorname{SigGNDeconv}(256,128,4,2,1)$ & $g 4^{1}$ \\
\hline$f 4^{1} \odot g 4^{1}$ & Upsample $(2,2)$ & $x 3$ \\
\hline$(x 3, f 3 \odot g 3)$ & $\operatorname{ResUnit}(256,64,3,1,1)$ & $f 3^{1}$ \\
\hline$f 4^{1} \odot g 4^{1}$ & $\operatorname{SigGNDeconv}(128,64,4,2,1)$ & $g 3^{1}$ \\
\hline$f 3^{1} \odot g 3^{1}$ & Upsample(2,2) & $x 2$ \\
\hline$(x 2, f 2 \odot g 2)$ & $\operatorname{ResUnit}(128,64,3,1,1)$ & $f 2^{1}$ \\
\hline$f 3^{1} \odot q 3^{1}$ & $\operatorname{SigGNDeconv}(128,64,4,2,1)$ & $g 2^{1}$ \\
\hline$f 2^{1} \odot g 2^{1}$ & Upsample $(2,2)$ & $x 1$ \\
\hline$(x 1, f 1 \odot g 1)$ & ResUnit $(128,64,3,1,1)$ & $f 1^{1}$ \\
\hline$f 2^{1} \odot g 2^{1}$ & $\operatorname{SigGNDeconv}(128,64,4,2,1)$ & $g 1^{1}$ \\
\hline$f 1^{1} \odot g 1^{1}$ & Upsample $(2,2)$ & $x 0$ \\
\hline$x 0$ & $\operatorname{ResUnit}(64,32,3,1,1)$ & $f 0^{1}$ \\
\hline$f 1^{1} \odot g 1^{1}$ & SigGNDeconv $(64,32,4,2,1)$ & $g 0^{1}$ \\
\hline$f 0^{1} \odot g 0^{1}$ & $\operatorname{Conv}(32,4,1,1,0)$ & $\left(\hat{\mathbf{A}}^{u v}, \sigma^{u v}\right)$ \\
\hline \multicolumn{2}{|c|}{ Model Complexity } & $11.7 \mathrm{M}$ \\
\hline
\end{tabular}
$\left(\mathbf{A}_{m}^{u v}, \mathbf{M}^{u v}\right)$. Outputs are the completed Albedo $\hat{\mathbf{A}}^{u v}$ and the uncertainty map $\sigma^{u v}$.

Table 8: Network architecture of the PyramidGAN discriminator $\mathcal{D}$.

\begin{tabular}{clc}
\hline Input & Layer & Output \\
\hline $\mathbf{I}_{g t} / \hat{\mathbf{I}}$ & SpectralConv $(3,32,4,2,1)+\mathrm{GN}(8)+\operatorname{LReLU}(.2)$ & $x 0$ \\
$x 0$ & SpectralConv $(32,64,4,2,1)+\mathrm{GN}(16)+\operatorname{LReLU}(.2)$ & $x 1$ \\
$x 1$ & SpectralConv $(64,1,1,1,0)$ & out 1 \\
$x 1$ & SpectralConv $(64,128,4,2,1)+\mathrm{GN}(32)+\operatorname{LReLU}(.2)$ & $x 2$ \\
$x 2$ & SpectralConv $(128,1,1,1,0)$ & out 2 \\
$x 2$ & SpectralConv $(128,256,4,2,1)+\mathrm{GN}(64)+\operatorname{LReLU}(.2)$ & $x 3$ \\
$x 3$ & SpectralConv $(256,1,1,1,0)$ & out3 \\
$x 3$ & SpectralConv $(256,512,4,2,1)+\mathrm{GN}(128)+\operatorname{LReLU}(.2)$ & $x 4$ \\
$x 4$ & SpectralConv(512, 1, 1,1,0) & out 4 \\
\hline \multicolumn{2}{c}{ Model Complexity } & $2.79 \mathrm{M}$ \\
\hline
\end{tabular}

- Albedo constancy loss is defined as:

$$
\begin{array}{r}
L_{\text {const }}(\mathbf{A})=\sum_{\mathbf{v}_{j}^{u v} \in \mathcal{N}_{i}} \omega\left(\mathbf{v}_{i}^{u v}, \mathbf{v}_{j}^{u v}\right) \| \mathbf{A}^{u v}\left(\mathbf{v}_{i}^{u v}\right)- \\
\mathbf{A}^{u v}\left(\mathbf{v}_{j}^{u v}\right) \|_{2}^{p},
\end{array}
$$

where $\mathcal{N}_{i}$ denotes the 4-neighborhood around $\mathbf{v}_{i}^{u v}$ and the weight $\omega\left(\mathbf{v}_{i}^{u v}, \mathbf{v}_{j}^{u v}\right)=\exp \left(-\alpha\left\|c\left(\mathbf{v}_{i}^{u v}\right)-c\left(\mathbf{v}_{j}^{u v}\right)\right\|\right)$ enforce 
Table 9: Network architecture of the face segmenter $\mathcal{S}$. $(x, y)$ represents the concatenation of tensors $x$ and $y$ along the channel dimension. The output of the network consist of a face mask $\mathbf{M}_{f}$, an occlusion mask $\mathbf{M}_{o}$ and a background mask $\mathbf{M}_{b}$.

\begin{tabular}{|c|c|c|}
\hline Input & Layer & Output \\
\hline Image & $\operatorname{ResUnit}(3,32,3,1,1)$ & $x 1$ \\
\hline$x 1$ & $\operatorname{ResUnit}(32,64,3,2,1)$ & $x 2$ \\
\hline$x 2$ & $\operatorname{ResUnit}(64,128,3,2,1)$ & $x 3$ \\
\hline$x 3$ & $\operatorname{ResUnit}(128,256,3,2,1)$ & $x 4$ \\
\hline$x 4$ & $\operatorname{ResUnit}(256,256,3,2,1)$ & $x 5$ \\
\hline$x 5$ & $\operatorname{ResUnit}(256,256,3,2,1)$ & $x 6$ \\
\hline$x 6$ & Upsample(2,2) & $x 5^{1}$ \\
\hline$\left(x 5^{1}, x 5\right)$ & ResUnit(512, 256, 3, 1, 1) & $x 5^{2}$ \\
\hline$x 5^{2}$ & Upsample(2,2) & $x 4^{1}$ \\
\hline$\left(x 4^{1}, x 4\right)$ & $\operatorname{ResUnit}(512,128,3,1,1)$ & $x 4^{2}$ \\
\hline$x 4^{2}$ & Upsample(2,2) & $x 3^{1}$ \\
\hline$\left(x 3^{1}, x 3\right)$ & $\operatorname{ResUnit}(256,64,3,1,1)$ & $x 3^{2}$ \\
\hline$x 3^{2}$ & Upsample(2,2) & $x 2^{1}$ \\
\hline$\left(x 2^{1}, x 2\right)$ & ResUnit(128, 32, 3, 1, 1) & $x 2^{2}$ \\
\hline$x 2^{2}$ & Upsample(2,2) & $x 1^{1}$ \\
\hline$\left(x 1^{1}, x 1\right)$ & $\operatorname{ResUnit}(64,32,3,1,1)$ & $x 1^{2}$ \\
\hline \multirow[t]{2}{*}{$x 1^{2}$} & $\operatorname{Conv}(32,3,1,1,0)+\operatorname{Softmax} 2 d$ & $\left(\mathbf{M}_{f}, \mathbf{M}_{o}, \mathbf{M}_{b}\right)$ \\
\hline & Model Complexity & $7.18 \mathrm{M}$ \\
\hline
\end{tabular}

that pixels with similar chromaticity should have similar albedo.

\section{C.3. Training Details}

3DMM Module: We train the 3DMM module in two stages. First, we train it using the 300W-3D dataset [53], which has ground-truth shape, pose, texture and landmark annotations, for $100 \mathrm{k}$ iterations in a supervised way. Then, we further train it on the CelebA dataset [24] with 1/10th of the original learning rate for further $30 \mathrm{k}$ iterations in an unsupervised way, whereby we use only the reconstruction loss, 2D landmark loss and the regularization losses. During this stage, we use landmark detections from HRNet [40] as groundtruth for the landmark loss. To make the 3DMM encoder robust to partial face images, we introduce artificial occlusions in the training images using random rectangular masks of varying sizes and locations. In addition, we also use random horizontal flipping as a data augmentation. During inference, occlusions are removed from the input image using the occlusion mask and passed through the 3DMM encoder to obtain occlusion-robust factorization.

Albedo Inpainting Module: The albedo inpainting module is trained on the CelebA dataset [24] for 30k iterations. To obtain the UV representations of the partial albedo and the mask, we re-project the 3D mesh obtained from the pretrained 3DMM module on the partial image and mask, respectively as shown in Main:Fig.3.b. On the GAN loss (Main:Eqn.2), we update the inpainter $\mathcal{G}$ and the discrimi- nator $\mathcal{D}$ alternatively using a ratio of $1: 1$. On all the other completion losses, we update the inpainter $\mathcal{G}$ continuously. Other than the random face masks, we use random horizontal flipping as the only data augmentation to train the albedo inpainter.

Face Segmentation Module: Since our method inpaints only the facial region in the UV domain, we restrict the image masks to lie on the face region too. For this, we train a UNet [31] based face segmentation model that separates the face region from the background, hair and inner mouth. The face segmenter predicts segmentation masks for (a) the face, (b) hair and other occlusions and (c) the background. We train the face segmentation module on the CelebAMask-HQ dataset [18] for a total of 50k iterations using the ground-truth annotations provided by the dataset. We use Focal loss [22] to train this module.

For all the modules, except the discriminator $\mathcal{D}$, we use the Adam optimizer with an initial learning rate of $10^{-4}$ and a step-decay of 0.98 per epoch, while for the PyramidGAN discriminator, we use an initial learning rate of $3 \times 10^{-4}$. The input images are first aligned to $256 \times 256$ using the method suggested in [18], which is the alignment used in the CelebA-HQ dataset. For training, we randomly crop the images to a size of $224 \times 224$ while during inference we use central crop. The full training takes 2 days on an Intel Xeon E5-2650 machine with two NVIDIA RTX 2080 GPUs, while inference takes $0.1 \mathrm{sec}$ per image on a single GPU.

\section{References}

[1] C. Barnes, E. Shechtman, A. Finkelstein, and D. B. Goldman. Patchmatch: A randomized correspondence algorithm for structural image editing. ACM Trans. Graph., 28(3):24, 2009.

[2] M. Bertalmio, G. Sapiro, V. Caselles, and C. Ballester. Image inpainting. In Proceedings of the 27th annual conference on Computer graphics and interactive techniques, pages 417424, 2000.

[3] V. Blanz and T. Vetter. A morphable model for the synthesis of $3 \mathrm{~d}$ faces. In Proceedings of the 26th annual conference on Computer graphics and interactive techniques, pages 187194, 1999.

[4] Y.-A. Chen, W.-C. Chen, C.-P. Wei, and Y.-C. F. Wang. Occlusion-aware face inpainting via generative adversarial networks. In 2017 IEEE International Conference on Image Processing (ICIP), pages 1202-1206. IEEE, 2017.

[5] D.-A. Clevert, T. Unterthiner, and S. Hochreiter. Fast and accurate deep network learning by exponential linear units (elus). arXiv preprint arXiv:1511.07289, 2015.

[6] A. Criminisi, P. Pérez, and K. Toyama. Region filling and object removal by exemplar-based image inpainting. IEEE Transactions on image processing, 13(9):1200-1212, 2004.

[7] J. Deng, S. Cheng, N. Xue, Y. Zhou, and S. Zafeiriou. Uvgan: Adversarial facial uv map completion for pose-invariant 
face recognition. In Proceedings of the IEEE conference on computer vision and pattern recognition, pages 7093-7102, 2018.

[8] B. Egger, S. Schönborn, A. Schneider, A. Kortylewski, A. Morel-Forster, C. Blumer, and T. Vetter. Occlusion-aware $3 \mathrm{~d}$ morphable models and an illumination prior for face image analysis. International Journal of Computer Vision, 126(12):1269-1287, 2018.

[9] B. Gecer, S. Ploumpis, I. Kotsia, and S. Zafeiriou. Ganfit: Generative adversarial network fitting for high fidelity $3 \mathrm{~d}$ face reconstruction. In Proceedings of the IEEE/CVF Conference on Computer Vision and Pattern Recognition (CVPR), June 2019.

[10] R. Gross, I. Matthews, J. Cohn, T. Kanade, and S. Baker. Multi-pie. Image and Vision Computing, 28(5):807-813, 2010.

[11] I. Gulrajani, F. Ahmed, M. Arjovsky, V. Dumoulin, and A. C. Courville. Improved training of wasserstein gans. In Advances in neural information processing systems, pages 5767-5777, 2017.

[12] J. Hays and A. A. Efros. Scene completion using millions of photographs. ACM Transactions on Graphics (ToG), 26(3):4-es, 2007.

[13] K. He, X. Zhang, S. Ren, and J. Sun. Deep residual learning for image recognition. In Proceedings of the IEEE conference on computer vision and pattern recognition, pages 770-778, 2016.

[14] S. Iizuka, E. Simo-Serra, and H. Ishikawa. Globally and locally consistent image completion. ACM Transactions on Graphics (ToG), 36(4):1-14, 2017.

[15] P. Isola, J.-Y. Zhu, T. Zhou, and A. A. Efros. Image-toimage translation with conditional adversarial networks. In Proceedings of the IEEE conference on computer vision and pattern recognition, pages 1125-1134, 2017.

[16] F. Juefei-Xu, R. Dey, V. N. Boddeti, and M. Savvides. Rankgan: a maximum margin ranking gan for generating faces. In Asian Conference on Computer Vision, pages 318. Springer, 2018.

[17] A. Kendall and Y. Gal. What uncertainties do we need in bayesian deep learning for computer vision? In Advances in neural information processing systems, pages 5574-5584, 2017.

[18] C.-H. Lee, Z. Liu, L. Wu, and P. Luo. Maskgan: Towards diverse and interactive facial image manipulation. In IEEE Conference on Computer Vision and Pattern Recognition (CVPR), 2020.

[19] X. Li, G. Hu, J. Zhu, W. Zuo, M. Wang, and L. Zhang. Learning symmetry consistent deep cnns for face completion. IEEE Transactions on Image Processing, 29:76417655, 2020.

[20] Y. Li, S. Liu, J. Yang, and M.-H. Yang. Generative face completion. In Proceedings of the IEEE conference on computer vision and pattern recognition, pages 3911-3919, 2017.

[21] Z. Li, Y. Hu, R. He, and Z. Sun. Learning disentangling and fusing networks for face completion under structured occlusions. Pattern Recognition, 99:107073, 2020.
[22] T.-Y. Lin, P. Goyal, R. Girshick, K. He, and P. Dollár. Focal loss for dense object detection. In Proceedings of the IEEE international conference on computer vision, pages 29802988, 2017.

[23] G. Liu, F. A. Reda, K. J. Shih, T.-C. Wang, A. Tao, and B. Catanzaro. Image inpainting for irregular holes using partial convolutions. In Proceedings of the European Conference on Computer Vision (ECCV), pages 85-100, 2018.

[24] Z. Liu, P. Luo, X. Wang, and X. Tang. Deep learning face attributes in the wild. In Proceedings of International Conference on Computer Vision (ICCV), December 2015.

[25] A. L. Maas, A. Y. Hannun, and A. Y. Ng. Rectifier nonlinearities improve neural network acoustic models. In Proc. icml, volume 30, page 3, 2013.

[26] T. Miyato, T. Kataoka, M. Koyama, and Y. Yoshida. Spectral normalization for generative adversarial networks. In International Conference on Learning Representations, 2018.

[27] D. Pathak, P. Krahenbuhl, J. Donahue, T. Darrell, and A. A. Efros. Context encoders: Feature learning by inpainting. In Proceedings of the IEEE conference on computer vision and pattern recognition, pages 2536-2544, 2016.

[28] P. Paysan, R. Knothe, B. Amberg, S. Romdhani, and T. Vetter. A 3d face model for pose and illumination invariant face recognition. In 2009 Sixth IEEE International Conference on Advanced Video and Signal Based Surveillance, pages 296301. Ieee, 2009.

[29] S. Ploumpis, E. Ververas, E. O’Sullivan, S. Moschoglou, H. Wang, N. Pears, W. Smith, B. Gecer, and S. P. Zafeiriou. Towards a complete $3 \mathrm{~d}$ morphable model of the human head. IEEE Transactions on Pattern Analysis and Machine Intelligence, 2020.

[30] R. Ramamoorthi and P. Hanrahan. An efficient representation for irradiance environment maps. In Proceedings of the 28 th annual conference on Computer graphics and interactive techniques, pages 497-500, 2001.

[31] O. Ronneberger, P. Fischer, and T. Brox. U-net: Convolutional networks for biomedical image segmentation. In International Conference on Medical image computing and computer-assisted intervention, pages 234-241. Springer, 2015.

[32] S. Sengupta, A. Kanazawa, C. D. Castillo, and D. W. Jacobs. Sfsnet: Learning shape, reflectance and illuminance of facesin the wild'. In Proceedings of the IEEE Conference on Computer Vision and Pattern Recognition, pages 62966305, 2018.

[33] A. Shocher, S. Bagon, P. Isola, and M. Irani. Ingan: Capturing and retargeting the" dna" of a natural image. In Proceedings of the IEEE/CVF International Conference on Computer Vision, pages 4492-4501, 2019.

[34] Z. Shu, E. Yumer, S. Hadap, K. Sunkavalli, E. Shechtman, and D. Samaras. Neural face editing with intrinsic image disentangling. In Proceedings of the IEEE conference on computer vision and pattern recognition, pages 5541-5550, 2017.

[35] L. Song, J. Cao, L. Song, Y. Hu, and R. He. Geometryaware face completion and editing. In Proceedings of the AAAI Conference on Artificial Intelligence, volume 33, pages 2506-2513, 2019. 
[36] A. Tewari, M. Zollhofer, H. Kim, P. Garrido, F. Bernard, P. Perez, and C. Theobalt. Mofa: Model-based deep convolutional face autoencoder for unsupervised monocular reconstruction. In Proceedings of the IEEE International Conference on Computer Vision Workshops, pages 1274-1283, 2017.

[37] L. Tran, F. Liu, and X. Liu. Towards high-fidelity nonlinear 3d face morphable model. In Proceedings of the IEEE Conference on Computer Vision and Pattern Recognition, pages 1126-1135, 2019.

[38] L. Tran and X. Liu. On learning 3d face morphable model from in-the-wild images. IEEE transactions on pattern analysis and machine intelligence, 2019.

[39] A. Tuán Trán, T. Hassner, I. Masi, E. Paz, Y. Nirkin, and G. Medioni. Extreme 3d face reconstruction: Seeing through occlusions. In Proceedings of the IEEE Conference on Computer Vision and Pattern Recognition, pages 3935-3944, 2018.

[40] J. Wang, K. Sun, T. Cheng, B. Jiang, C. Deng, Y. Zhao, D. Liu, Y. Mu, M. Tan, X. Wang, et al. Deep high-resolution representation learning for visual recognition. IEEE transactions on pattern analysis and machine intelligence, 2020.

[41] T.-C. Wang, M.-Y. Liu, J.-Y. Zhu, A. Tao, J. Kautz, and B. Catanzaro. High-resolution image synthesis and semantic manipulation with conditional gans. In Proceedings of the IEEE conference on computer vision and pattern recognition, pages 8798-8807, 2018.

[42] S. Wu, C. Rupprecht, and A. Vedaldi. Unsupervised learning of probably symmetric deformable $3 \mathrm{~d}$ objects from images in the wild. In Proceedings of the IEEE/CVF Conference on Computer Vision and Pattern Recognition, pages 1-10, 2020.

[43] Y. Wu and K. He. Group normalization. In Proceedings of the European conference on computer vision (ECCV), pages 3-19, 2018.

[44] R. A. Yeh, C. Chen, T. Yian Lim, A. G. Schwing, M. Hasegawa-Johnson, and M. N. Do. Semantic image inpainting with deep generative models. In Proceedings of the IEEE conference on computer vision and pattern recognition, pages 5485-5493, 2017.

[45] J. Yu, Z. Lin, J. Yang, X. Shen, X. Lu, and T. S. Huang. Generative image inpainting with contextual attention. In Proceedings of the IEEE conference on computer vision and pattern recognition, pages 5505-5514, 2018.

[46] J. Yu, Z. Lin, J. Yang, X. Shen, X. Lu, and T. S. Huang. Free-form image inpainting with gated convolution. In Proceedings of the IEEE International Conference on Computer Vision, pages 4471-4480, 2019.

[47] X. Yuan and I. K. Park. Face de-occlusion using 3d morphable model and generative adversarial network. In Proceedings of the IEEE International Conference on Computer Vision, pages 10062-10071, 2019.

[48] J. Zhang, R. Zhan, D. Sun, and G. Pan. Symmetry-aware face completion with generative adversarial networks. In Asian Conference on Computer Vision, pages 289-304. Springer, 2018.
[49] R. Zhang, P. Isola, A. A. Efros, E. Shechtman, and O. Wang. The unreasonable effectiveness of deep features as a perceptual metric. In CVPR, 2018.

[50] S. Zhang, R. He, Z. Sun, and T. Tan. Demeshnet: Blind face inpainting for deep meshface verification. IEEE Transactions on Information Forensics and Security, 13(3):637-647, 2017.

[51] C. Zheng, T.-J. Cham, and J. Cai. Pluralistic image completion. In Proceedings of the IEEE Conference on Computer Vision and Pattern Recognition, pages 1438-1447, 2019.

[52] T. Zhou, C. Ding, S. Lin, X. Wang, and D. Tao. Learning oracle attention for high-fidelity face completion. In Proceedings of the IEEE/CVF Conference on Computer Vision and Pattern Recognition, pages 7680-7689, 2020.

[53] X. Zhu, Z. Lei, X. Liu, H. Shi, and S. Z. Li. Face alignment across large poses: A $3 \mathrm{~d}$ solution. In Proceedings of the IEEE conference on computer vision and pattern recognition, pages 146-155, 2016. 